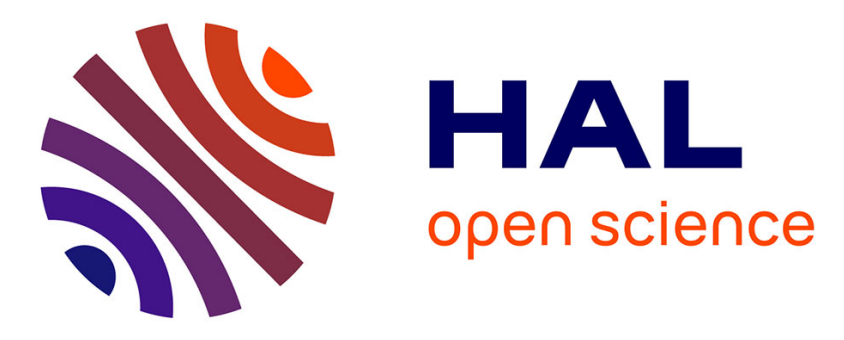

\title{
Denoising of PET images by combining wavelets and curvelets for improved preservation of resolution and quantitation.
}

Adrien Le Pogam, Houda Hanzouli, Mathieu Hatt, Catherine Cheze Le Rest, Dimitris Visvikis

\section{To cite this version:}

Adrien Le Pogam, Houda Hanzouli, Mathieu Hatt, Catherine Cheze Le Rest, Dimitris Visvikis. Denoising of PET images by combining wavelets and curvelets for improved preservation of resolution and quantitation.. Medical Image Analysis, 2013, 17 (8), pp.877-891. 10.1016/j.media.2013.05.005 . inserm-00844926

\section{HAL Id: inserm-00844926 https://www.hal.inserm.fr/inserm-00844926}

Submitted on 16 Jul 2013

HAL is a multi-disciplinary open access archive for the deposit and dissemination of scientific research documents, whether they are published or not. The documents may come from teaching and research institutions in France or abroad, or from public or private research centers.
L'archive ouverte pluridisciplinaire HAL, est destinée au dépôt et à la diffusion de documents scientifiques de niveau recherche, publiés ou non, émanant des établissements d'enseignement et de recherche français ou étrangers, des laboratoires publics ou privés. 


\title{
Denoising of PET images by combining wavelets and curvelets for improved preservation of resolution and quantitation
}

\author{
A. Le Pogam ${ }^{A}$, H. Hanzouli ${ }^{A}$, M. Hatt ${ }^{A^{*}}$, C. Cheze Le Rest ${ }^{B}$, D. Visvikis ${ }^{A}$ \\ A INSERM, UMR 1101, LaTIM, CHRU Morvan, Brest, France

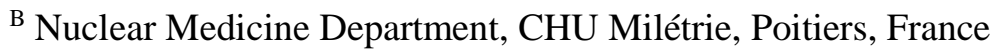 \\ ${ }^{*}$ Corresponding author: \\ Mathieu Hatt \\ INSERM, UMR 1101, LaTIM \\ CHRU Morvan \\ 5 avenue Foch \\ 29609 Brest Cedex \\ France \\ Tel: +33298018111 \\ Fax: +33298018124 \\ Email: hatt@univ-brest.fr
}

\begin{abstract}
Denoising of Positron Emission Tomography (PET) images is a challenging task due to the inherent low signal-to-noise ratio (SNR) of the acquired data. A pre-processing denoising step may facilitate and improve the results of further steps such as segmentation, quantification or textural features characterization. Different recent denoising techniques have been introduced and most state-of-the-art methods are based on filtering in the wavelet domain. However, the wavelet transform suffers from some limitations due to its non optimal processing of edge discontinuities. More recently, a new multi scale geometric approach has been proposed, namely the curvelet transform. It extends the wavelet transform to account for directional properties in the image. In order to address the issue of resolution loss associated with standard denoising, we considered a strategy combining the complementary wavelet and curvelet transforms. We compared different figures of merit (e.g. SNR increase, noise decrease in homogeneous regions, resolution loss, and intensity bias) on simulated and clinical datasets with the proposed combined approach and the wavelet-only and curvelet-only filtering techniques. The three methods led to an increase of the SNR. Regarding the quantitative accuracy however, the wavelet and curvelet only denoising approaches led to larger biases in the intensity and the contrast than the proposed combined algorithm. This approach could become an alternative solution to filters currently used after image reconstruction in clinical systems such as the Gaussian filter.
\end{abstract}

Keywords: positron emission tomography; curvelet transform; wavelet transform; denoising; filtering. 


\section{INTRODUCTION}

Statistical analysis and quantitative evaluation of Positron Emission Tomography (PET) images often involve preprocessing steps. As PET images are of low signal-to-noise ratio (SNR), the most common one consists of denoising, which is usually carried out via Gaussian filtering, as sometimes implemented in iterative image reconstruction algorithms (Alessio and Kinahan 2006). However, although Gaussian filtering may significantly increase the SNR, it also smoothes the image, altering the mean signal in heterogeneous regions. It also blurs contours, which is to be clearly avoided in PET imaging that is already characterized by limited spatial resolution and associated partial volume effects. PET denoising approaches based on filtering in the spatial frequency domain through wavelet transform (WT) have been reported to improve the qualitative visual aspect and quantitation of images in a wide variety of applications (Turkheimer et al. 2000; Shih et al. 2005; Alpert et al. 2006) and are still under investigation in recent publications (Shidahara et al. 2007; Turkheimer et al. 2008; Boussion et al. 2009; Shalchian et al. 2009).

The WT was initially introduced as a mathematical tool for signal processing with non-stationary behavior (Mallat 1989). In PET imaging, the WT can be applied to the spatial distribution of the radiotracer after reconstruction and can convert the original voxel value distribution into a multi scale representation. One of the relevant properties of WT is its ability to obtain a sparse representation of the initial signal while retaining a homogeneous noise distribution. For a denoising application, this separation can be exploited to increase the SNR by filtering out the noise in the wavelet domain. The sparser the transformation, the more efficient is its use for denoising. Whereas the 1D WT demonstrates good performance at representing point singularities, it is not the case anymore in higher dimensions due to the lack of geometrical property of wavelets. The 2D or 3D WT consequently provides a suboptimal representation of images with highly anisotropic elements such as curvilinear structures (e.g. edges) (Easley et al. 2008). Discontinuities across such structures affect all the wavelet coefficients on this structure and the denoising process yields overly smoothed results. To address this issue, a wide variety of alternative approaches to image decomposition have appeared in recent years, initiated by the ridgelet transform (RT) (Cands 1998). More recently, a new analysis technique called the curvelet transform (CT) has been developed (Candès and Donoho 2002; Candes 2006), improving the properties of the ridgelet approach. Image representation via curvelets is built upon multi-scale analysis and geometry, with curvelets taking the form of highly anisotropic elements with high directional sensitivity. This representation allows additional geometrical information and consequently leads to a more accurate image representation than through WT, as illustrated in figure 1. Although recently introduced and still under development, CT has already generated interesting results in a wide range of image processing applications including denoising (Starck et al. 2002; Hyder and Sukanesh 2011). However, CT does not lead to an optimal sparse representation of the isotropic parts of the image compared to WT, and consequently denoising based on filtering in the curvelet domain (Curvelet denoising, CD) does not perform as well as wavelet denoising (WD) (Mallat 2009; Starck et al. 2010). Therefore, a combined approach exploiting the advantages provided by both techniques may potentially lead to improved performance. 


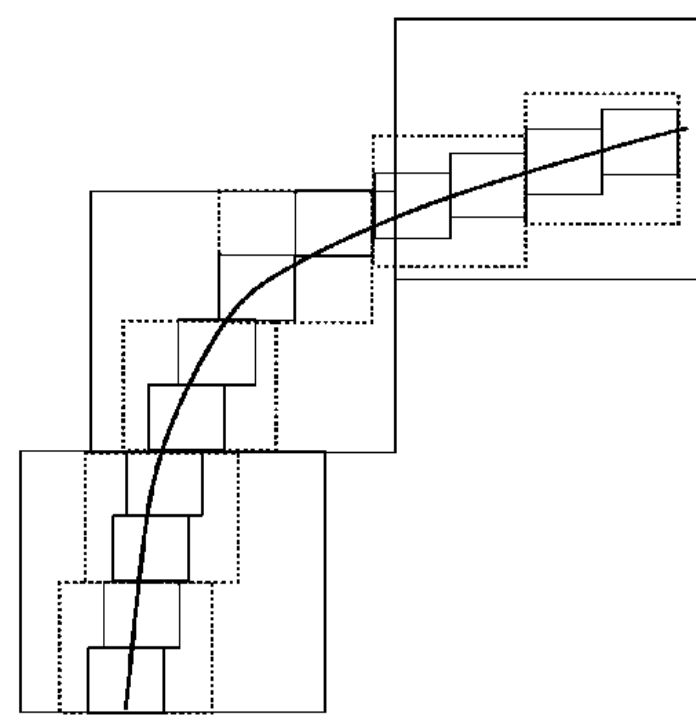

(a)

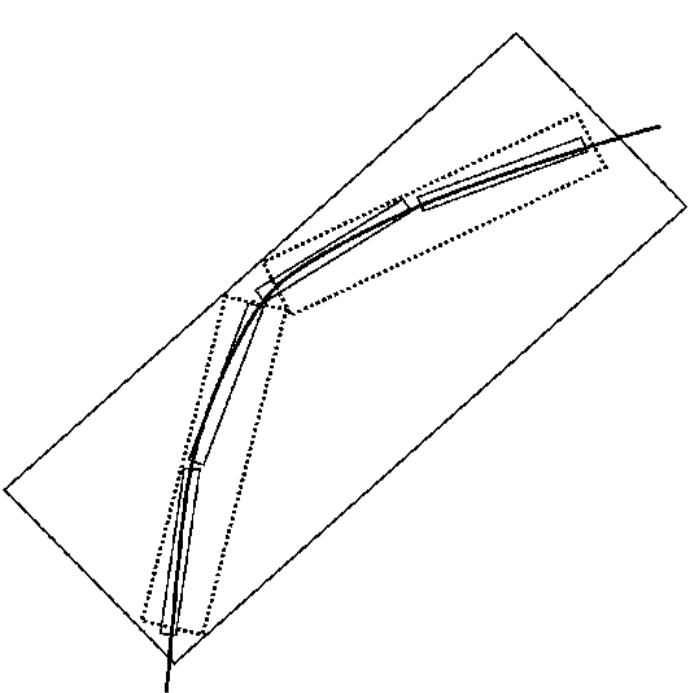

(b)

Figure 1: 2D decomposition of an edge via (a) the wavelet and (b) the curvelet transforms

In this work, we propose a new methodology to filter the noise in 3D PET images using a combined implementation of the 3D isotropic undecimated wavelet transform (IUDWT) (Starck et al. 2007) and the 3D discrete curvelet transform (DCT) (Starck et al. 2002), incorporating a thresholding of the coefficients based on a local adaptive analysis (Sendur and Selesnick 2002). This approach will be referred to from here onwards as wavelet-curvelet denoising (WCD). The objective and principle of this combination is to recover (thanks to CD) the edges that have been discarded by the WD step from the residual image (the difference between the original image and the WD image), and to incorporate them back into the WD PET image. The idea of combining several such representations is not new (Huo 1999; Chen 2001), however, in most cases, the proposed algorithms are computationally very expensive. The methodology proposed here consists in ensuring that the reconstruction incorporates information judged as significant by any of the two different wavelet and curvelet representations. One alternative approach consists in combining WD and CD, performed in parallel on the initial noisy image (Starck 2001). This approach is not straightforward, because contrary to wavelet coefficients, curvelets coefficients cannot be easily spatially localized in the original image due to the various steps involved in their calculation. A more straightforward and easier approach, previously proposed within the context of medical image segmentation, consists in applying a CD on the residual image obtained from WD (Shen 2006). This method only considered, however the use of basic wavelet and curvelet transforms, and its potential interest for image denoising or PET imaging has never been investigated. The main objective of this work was therefore the evaluation of such a straightforward combined wavelet and curvelet denoising for PET imaging compared with independent WD or CD schemes. To the best of our knowledge, this has not been previously considered for PET images denoising purposes, although it presents promising properties. It is especially able to retain relevant details information (isotropic as well as curvilinear structures) while discarding local noise. The ability of each method to preserve local contrast and quantitation while improving SNR was investigated on several simulated and clinical datasets. 


\section{MATERIALS AND METHODS}

The main assumption in the denoising process is that the observed data I contains the true signal $\mathrm{S}$ with additive Gaussian noise N such as:

$$
\mathrm{I}=\mathrm{S}+\mathrm{N}
$$

Although raw PET data follow Poisson distribution, the noise in reconstructed PET images using iterative algorithms cannot be considered Poisson and a Gaussian assumption can be made (Alessio and Kinahan 2006; Hatt et al. 2009). Denoising then consists of the three following steps:

$$
\begin{aligned}
& \mathrm{Y}=A(\mathrm{I}) \\
& \mathrm{Z}=\mathrm{T}\left(\mathrm{Y}_{\mathrm{t}}\right) \\
& \mathrm{S}_{\mathrm{est}}=A^{-1}(Z)
\end{aligned}
$$

where $\mathrm{A}$ and $\mathrm{A}^{-1}$ are the direct and inverse transform operators and $\mathrm{T}(\mathrm{t})$ the denoising operator depending on the threshold value t. Details regarding implementation of the operator $\mathrm{T}$ or selection of the threshold $\mathrm{t}$ are covered in the following sections.

\subsection{Wavelet transform (WT)}

Among the various available algorithms performing discrete wavelet transforms of an image, a common approach widely used in PET imaging is the Isotropic Undecimated Wavelet Transform (IUWT) implemented using the 'à trous' algorithm (Dutilleux 1987). This translation invariance inducing methodology is of particular interest when investigating image denoising. The non decimation makes the decomposition redundant and allows avoiding pseudo Gibbs phenomenon after reconstruction. The straightforward and fast implementation without loss of any kind, as well as the easy navigation between the different scales and the isotropic process are among the other practical advantages of this methodology. We therefore used the "à trous" algorithm to perform the WT (Starck et al. 2007), more specifically a 3D extension of this algorithm based on a binomial filter of order 4 (Le Pogam et al. 2011). In this work, the first two layers were generated and then filtered in a subsequent step by the denoising method described in the following subsection.

\subsection{Wavelet-based denoising (WD)}

A first approach for WD was called Visushrink (Donoho and Johnstone 1994), in which a general threshold (now usually denoted "Universal threshold") is defined requiring the standard deviation (SD) $\sigma$ of the WT values. In most common cases, it is impossible to measure $\sigma$ from the noisy image, but it is possible to estimate it from the first subband of the WT using equation 3 where $\mathrm{w}_{\mathrm{ij}}$ are WT coefficients of the first sub-band (Donoho and Johnstone 1994):

$$
\sigma=\operatorname{Median}\left(\left|w_{i j}\right|\right) / 0.6745
$$


The main practical interest of this approach is its easy implementation, although more powerful denoising techniques have been developed. Among these, most of the wavelet-based thresholding methods such as BayesShrink (Chang et al. 2000) or SureShrink (Donoho and Johnstone 1995) assume that wavelet coefficients are independent. The SureShrink approach purposely devised for PET (Turkheimer et al. 1999) is considered as a state-of-the-art denoising. However, the assumption of independence of the wavelet coefficients is questionable and the performance of WD can be significantly improved taking into account the statistical relationships between wavelet coefficients (Crouse et al. 1998; Simoncelli 1999; Sendur and Selesnick 2002). A new shrinkage function, depending on both the wavelet coefficient $\mathrm{w}_{1}$ and its parent $\mathrm{w}_{2}$ (which is at the same spatial position as $\mathrm{w}_{1}$ but at the next coarser scale), was introduced (Sendur and Selesnick 2002). The following assumptions regarding the observed data are considered:

$$
\mathrm{y}=\mathrm{w}+\mathrm{n}
$$

with, $\mathrm{w}=\left(\mathrm{w}_{1}, \mathrm{w}_{2}\right), \mathrm{y}=\left(\mathrm{y}_{1}, \mathrm{y}_{2}\right)$, and $\left(\mathrm{n}_{1}, \mathrm{n}_{2}\right)$ independent and identically distributed zero mean Gaussian with variance

$\sigma_{n}^{2}$ estimated by equation 3. The following simple non-Gaussian bivariate probability distribution function (pdf) was introduced to model the statistics of wavelet coefficients and capture the dependence between a given wavelet coefficient and its parent (Sendur and Selesnick 2002):

$$
p_{w}(w)=3 / 2 \pi \sigma^{2} \exp \left(-\sqrt{3} / \sigma \sqrt{w_{1}^{2}+w_{2}^{2}}\right)
$$

Using Bayesian estimation theory with the maximum a posteriori estimator of $\mathrm{w}_{1}$, a simple non-linear shrinkage function (called BiShrink) can be derived from this model and generalizes the soft thresholding approach (Donoho 1995):

$$
\hat{w}_{1}=\left|\sqrt{y_{1}^{2}+y_{2}^{2}}-\sqrt{3} \sigma_{n}^{2} / \sigma\right|+/ \sqrt{y_{1}^{2}+y_{2}^{2}} y_{1}
$$

For this function, the smaller the parent value the greater the shrinkage, which is consistent with other models. In this study, we used an extended local adaptive version in which $\sigma$ is estimated using a neighborhood (Sendur and Selesnick 2002). We investigated the potential impact of five different neighborhood sizes (from 3 to 13 voxels), as recommended (Sendur and Selesnick 2002).

\subsection{Curvelet transform $(C T)$}

For the analysis of data containing anisotropic features, wavelets are no longer optimal. This has motivated the development of new multi scale decompositions such as the curvelet transform (Candès and Donoho 2002; Starck et al. 2002; Candes 2006). The first generation CT (Starck et al. 2002) used a series of steps involving the ridgelet analysis and the radon transform of an image. The algorithm has been updated and the use of ridgelet was discarded to reduce redundancy and computational expense (Candes 2006). CT is considered as a multi scale and multi directional transform with elements indexed by position, scale and direction parameters, with the shape of tight frames co-localized 
in the spatial and frequency domains. Although the CT presents similar localization properties (in the spatial and frequency domains) compared to the WT, it is associated with higher degrees of directionality and anisotropy. Since one step of the ridgelet algorithm is based on the WT and as the redundancy property can be of interest for denoising purposes, the second generation CT (Candes 2006) was not the best choice for our comparison study. We consequently decided to use the first generation discrete CT (Starck et al. 2002) based on an implementation of the 3D Discrete Analytical Ridgelet Transform (3D DART) (Helbert et al. 2006). This 3D implementation allows an exact reconstruction in the same way as for the WT. The idea of the discrete CT is to decompose the image into a set of wavelet bands and to analyze each band by a local ridgelet transform with a different block size for each scale level. Figure 2 illustrates the edge decomposition via the first generation's CT.

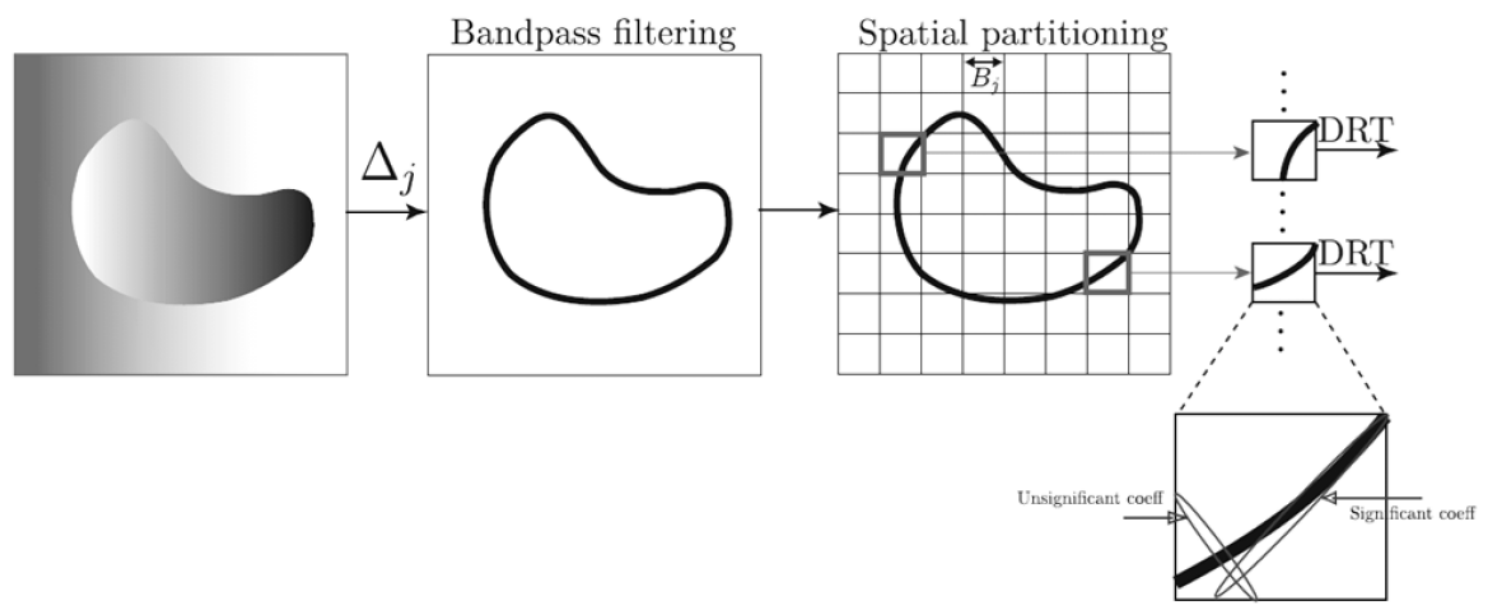

Figure 2: Decompositions of an edge via the first generation CT

The discrete CT of a continuous function $f\left(x_{1}, x_{2}\right)$ makes use of a dyadic sequence of scales and a bank of filters $\left(P_{0} f, \Delta_{1} f, \Delta_{2} f, \ldots\right)$.

$$
\begin{gathered}
\text { with } \quad \Delta_{s}=\psi_{2 s} \times f \\
\hat{\psi}_{2 s}(\xi)=\hat{\psi}_{2 s}\left(2^{-2 s} \xi\right) \\
\text { and } \hat{\psi}(2 v)=\hat{\phi}(v)-\hat{\phi}(2 v)
\end{gathered}
$$

with $\hat{\phi}$ the scaling function.

The decomposition can then be summed up by the following steps which are also illustrated in figure 3:

1) Sub-band decomposition of an object f:

$$
f \mapsto\left(P_{0} f, \Delta_{1} f, \Delta_{2} f, \ldots\right) .
$$

2) Smooth Partitioning: each sub-band is smoothly windowed into a set of dyadic squares $\mathcal{Q}_{s}$.

$$
\Delta f \mapsto\left(w_{Q} \Delta_{s} f\right)_{Q \in Q_{s}}
$$


3) Local Ridgelet Analysis: each window is analyzed via the 3D local DART (see the Appendix).

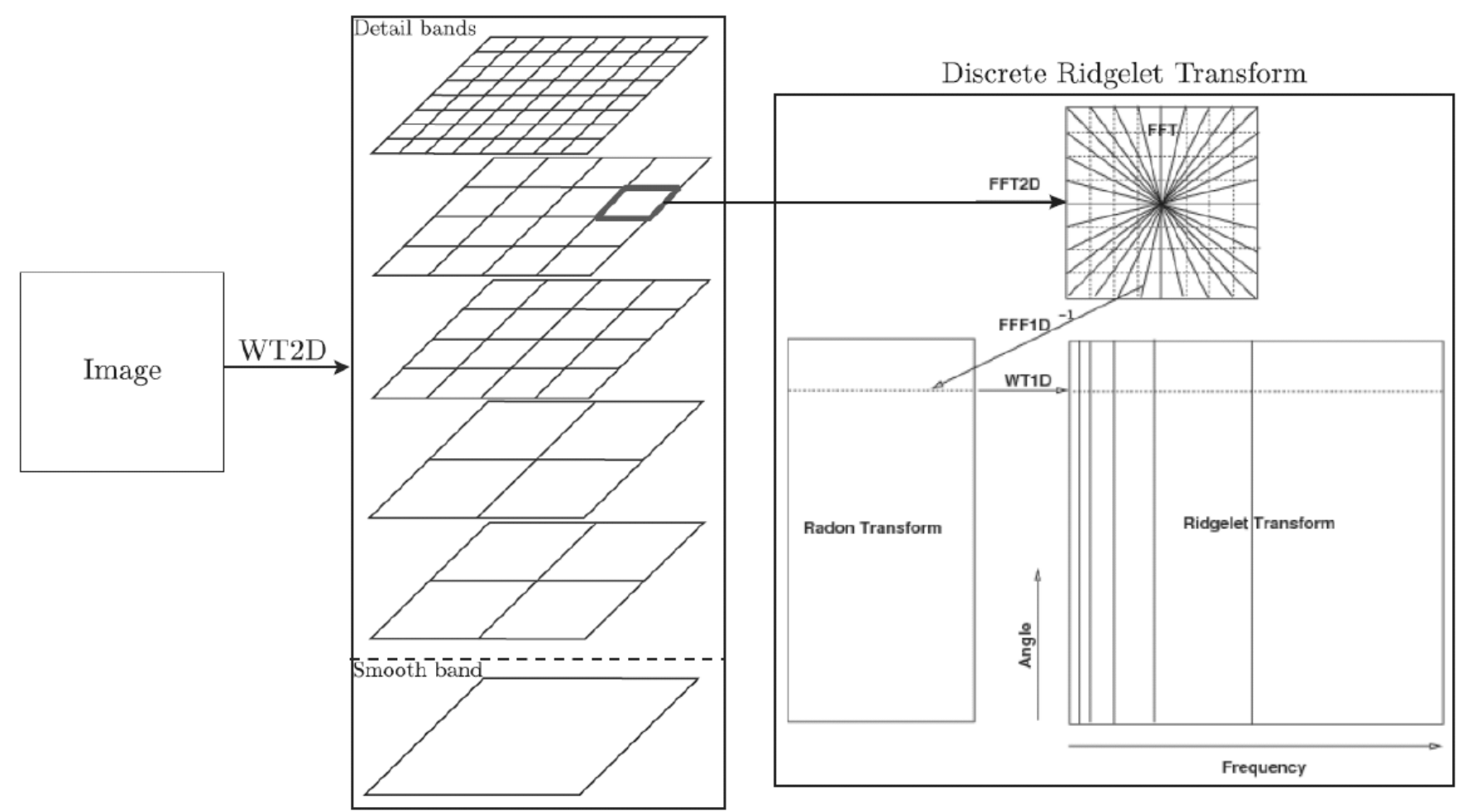

Figure 3: Flowchart for the algorithm used to obtain first generation CT

\subsection{Curvelet-based denoising $(C D)$}

As described in the previous paragraph, the CT process is based on WT. During the local ridgelet analysis step, each line in the ridgelet transform image corresponds to 1D wavelet coefficients obtained from the Radon transform. These coefficients can consequently be processed by the same WD process (Sendur and Selesnick 2002) as the one previously described for 3D images in the wavelet-based denoising (see figure 3). Similarly to the 3D analysis, a comparison using different window sizes $(3,5,7,11$ and 13$)$ was performed.

\subsection{Combined denoising scheme}

As previously explained, WT and CT exhibit different behaviors. Wavelets do not perfectly restore anisotropic structures (such as edges) whereas curvelets do not correctly handle small isotropic elements. These transforms therefore exhibit complementary properties and may consequently be combined within an improved denoising strategy. The proposed WCD methodology, illustrated in figure 4, consists in improving the current state-of-the-art WD method by analyzing its residual image $(\mathrm{R})$ by $\mathrm{CD}$. The residual R contains both the noise and the structures (mainly anisotropic such as edges) lost during the WD process. These structures can consequently be picked up from R via CD based on the edge-preserving property of the CT, and then inserted back into the initial noisy image processed by WD. 
Again, the optimal neighboring window size was assessed by comparing the different values $(3,5,7,11$ and 13$)$. The combined WCD methodology was implemented using the $\mathrm{C}++$ language on a Pentium 4, single processor, $2 \mathrm{~Gb}$ memory PC. Denoising of a $128 \times 128 \times 128$ volume requires less than 5 minutes and this time could be easily reduced by parallel computing.

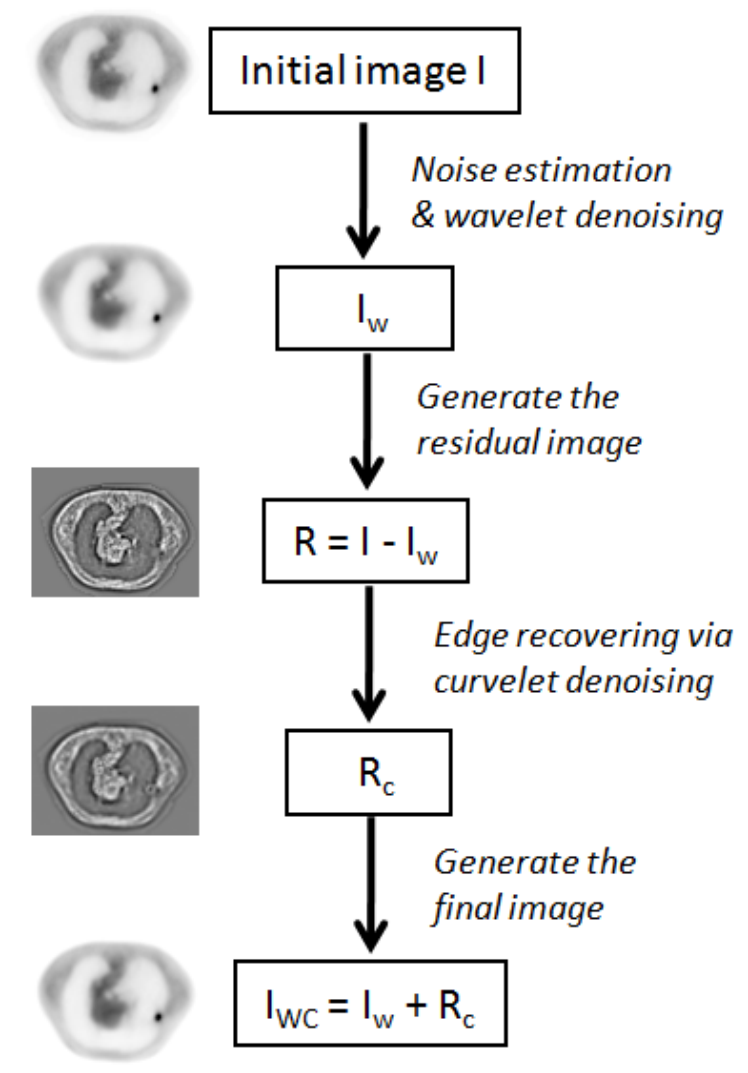

Figure 4: Combined Wavelet-Curvelet denoising (WCD) method flow chart

\subsection{Evaluation study}

We chose to build a clinically-relevant dataset of images to compare the three approaches by considering simulated and clinical PET images in the neurology and oncology domains, obtained with different PET scanners and reconstruction processes. All our realistic simulations, based on our previous works (Lamare et al. 2006; Le Maitre et al. 2009), were consistent in terms of parameters of acquisition and reconstruction compared to true clinical PET acquisitions. For all datasets, the three denoising schemes (WD, CD and the proposed WCD) were compared in terms of SNR improvements as well as contrast loss and intensity biases (for more details, see 2.6.3 quantitative analysis). For one of the datasets, the results obtained with WD, CD and WCD were in addition compared with a Gaussian filter. All datasets are illustrated in figure 5 and described in the following sections.

\subsubsection{Simulated datasets}

\subsubsection{Uniform cylinder}

The performance of the WCD was first evaluated using a realistic simulation of a ${ }^{18} \mathrm{~F}$ filled homogeneous (uniform 
activity concentration of $1 \mathrm{~Bq} / \mathrm{mm}^{3}$ ) cylindrical phantom (radius $120 \mathrm{~mm}$, length $180 \mathrm{~mm}$ ). Although this phantom is extremely simple, it allows the use of a method dedicated to noise estimation (Lodge et al. 2010) (more details in section 2.6.3). The acquisition with a Philips Gemini PET scanner was simulated using the Monte Carlo-based simulator Geant4 Application for Tomography Emission (GATE) allowing the modeling of all relevant physical effects of a real PET acquisition (positron annihilation, photoelectric effect, and Compton scattering) (Lamare et al. 2006). A total of respectively 5, 10, 15, 20 and 25 million coincidences were generated. Images were reconstructed using the One Pass Listmode Expectation Maximization (OPL-EM) algorithm (Reader et al. 2002) and corrected for attenuation. The final reconstructed images (illustrated in figure $5 \mathrm{a}$ ) consisted of volumes of $141 \times 141 \times 45$ voxels of $4 \times 4 \times 4 \mathrm{~mm}^{3}$.

\subsubsection{IEC phantom}

A second dataset consisted of simulations of the IEC phantom (Jordan 1990), an homogeneous cylinder similar to the previous phantom but containing six spheres of different diameters $\left(10,13,17,22,28\right.$ and 37mm) and filled with ${ }^{18} \mathrm{~F}$. Acquisitions with the Philips Allegro PET scanner were simulated using GATE. A total of respectively 20, 40 and 60 millions coincidences were generated considering sphere-to-cylinder activity concentration ratios of 4:1 and 8:1. Images were reconstructed using the OPL-EM algorithm ( 7 iterations, 1 subset) and corrected for attenuation, with no post-filtering, allowing the comparison with the standard clinical Gaussian filter, which was applied using a 3D FWHM of $5 \mathrm{~mm}$. Two different sizes of the voxel grid $(64 \times 64 \times 64$ and $128 \times 128 \times 128)$ were in addition considered in the reconstruction leading to voxels of $4 \times 4 \times 4$ and $2 \times 2 \times 2 \mathrm{~mm}^{3}$. Twelve different configurations were therefore analyzed. Figure $5 \mathrm{~b}$ illustrates this dataset for the case $8: 1,4 \times 4 \times 4 \mathrm{~m}^{3}, 60$ million coincidences.

\subsubsection{Brain phantom}

The last simulated dataset was an image generated using a segmented brain phantom based on measured T1 MRI images (Zubal et al. 1994). A functional static ECAT HR $+{ }^{18}$ F-FDG PET image was generated using the procedure

fully described previously (Tsoumpas et al. 2008; Le Pogam et al. 2011). Clinically measured plasma time activity curves (TACs) were used to generate a set of TACs for each segmented anatomical region of the brain phantom according to 28 different clinical dynamic frames $(1 \times 30 s, 1 \times 15 s, 1 \times 5 s, 4 \times 10 s, 4 \times 30 s, 4 \times 60 s, 4 \times 120 s, 9 \times 300 s)$. Pathological parameters were also introduced in the parietal and the anterior frontal lobes. Projection data of the ECAT $\mathrm{HR}+$ scanner were then generated from these dynamic images, and corrected for attenuation, normalization, scatter and randoms. Poisson noise was finally added to the sinograms. Images were subsequently reconstructed by filtered back projection (FBP) and the static PET image was obtained by summing the last six temporal frames. This simulated static brain PET acquisition and its corresponding ground-truth are illustrated in figure 5c.

\subsubsection{Clinical datasets}

The algorithms were also applied to two different sets of lung cancer patients whole-body ${ }^{18} \mathrm{~F}-\mathrm{FDG}$ PET/CT images (10 in each dataset). 


\subsubsection{Large voxels and post reconstruction smoothing}

The first dataset images were acquired on a GE Discovery STE 4-slice PET/CT, 55 min after injection of $355 \mathrm{MBq}$ of FDG, with 3 min per bed position. PET images were corrected for attenuation using CT and reconstructed using $4.68 \times 4.68 \times 3.27 \mathrm{~mm}^{3}$ voxels $(128 \times 128$ matrix $)$ with the OSEM algorithm using standard clinical protocol parameters ( 2 iterations, 28 subsets), and a post reconstruction Gaussian filter with a 5mm FWHM, resulting in rather smoothed images. One example of the first dataset is illustrated in figure $5 \mathrm{~d}$.

\subsubsection{Small voxels and no post reconstruction smoothing}

Acquisitions were performed on a GE Discovery LS PET/CT scanner, 60 min after injection of $350 \mathrm{MBq}$ of FDG, with 3 min per bed position. PET images were reconstructed using $1.95 \times 1.95 \times 4.25 \mathrm{~mm}^{3}$ voxels $(256 \times 256$ matrix $)$ with the OSEM algorithm, using standard clinical protocol parameters (2 iterations, 28 subsets) with a CT-based attenuation correction and no post-filtering. These images had a different "salt and pepper" aspect and contained a higher level of noise compared to the first dataset. One example is illustrated in figure 5e.

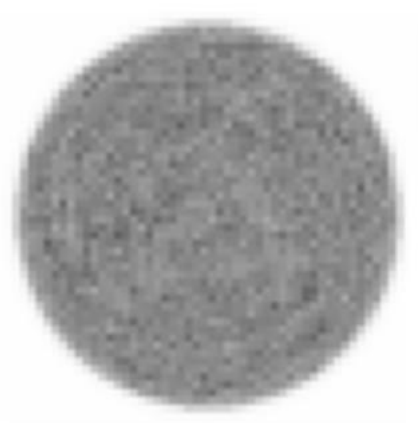

(a)

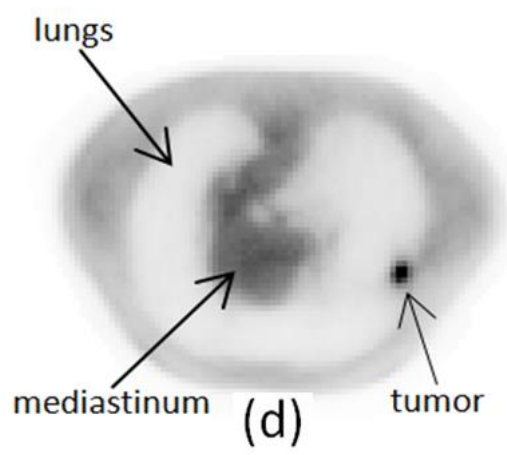

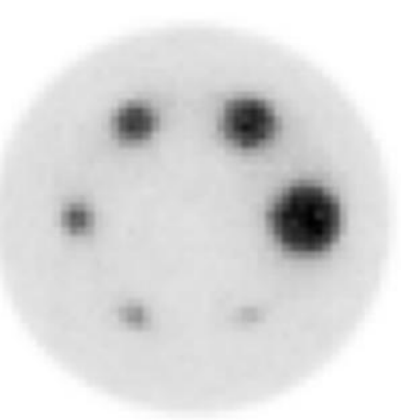

(b)

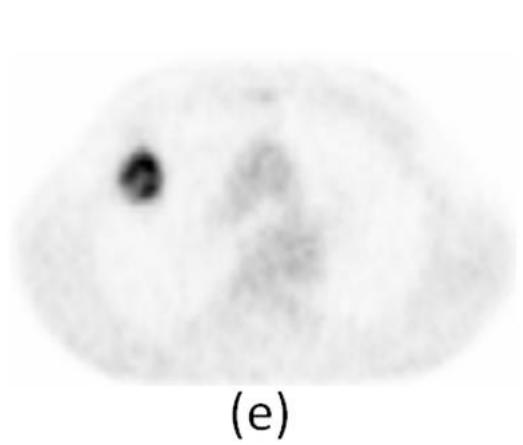

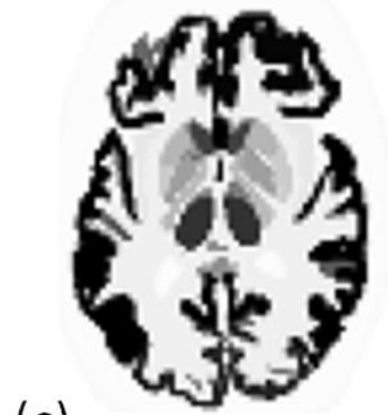

(c)

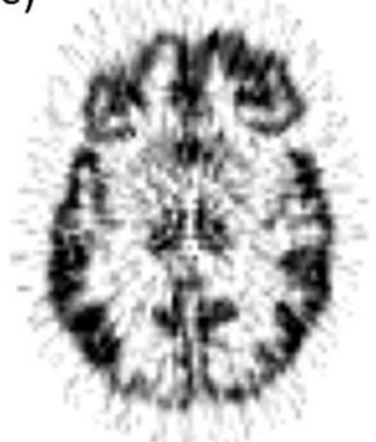

Figure 5: Illustration of the various datasets used in this study: uniform simulated cylinder (20 million coincidences) (a), IEC phantom simulation $\left(4 \times 4 \times 4 \mathrm{~mm}^{3}, 60\right.$ millions coincidences, 8:1 ratio) (b), brain phantom with ground truth (up) and simulated image below (c), first clinical dataset (d), second clinical dataset (e).

\subsubsection{Quantitative analysis}

For the first simulated dataset of the uniformly filled cylinder (figure 5a), we used a technique for SNR measurements and only possible with a uniform phantom acquisition (Lodge et al. 2010). In this approach, noise is measured by determining the difference between corresponding voxels in two acquisitions with the same conditions and by calculating the SD of these differences. This corresponds to a noise analysis with multiple realizations as it is assumed that each pair of slices consists of independent noise observations (Lodge et al. 2010). For one single simulation we 
therefore obtained $\mathrm{n}$ multiple realizations where $\mathrm{n}$ is the number of slices.

For all other datasets, manually drawn regions of interest (ROI) were placed over identified structures (spheres, tumors) and within large homogeneous regions (such as the lungs or the liver for the clinical images). Quantitative measurement of SNR was extracted from these ROIs on the images using respectively WD, CD and the proposed WCD methodologies (and Gaussian filtering in the case of the simulated IEC phantom). The SNR was defined according to equation (12) (Webb 2003):

$$
S N R=20 \log _{10}\left(m_{i} / \sigma_{i}\right) d B
$$

with $\mathrm{m}_{\mathrm{i}}$ and $\sigma_{i}$ the mean and SD of the voxels' intensities within the ROIs, evaluated before and after the denoising step.

To evaluate the potential loss of resolution induced by the denoising and the preservation of the information regarding structures of interest, we used line profiles through various regions. In addition, the mean contrast along these intensity profiles across tissue boundaries (chosen as going from one uniform area outside the chosen ROI to another one inside the ROI) was also calculated in order to assess the amount of smoothness introduced by the denoising step using the following formula:

$$
\text { Contrast }=100 / L \times \sum_{i=0}^{L-1}\left|x_{i+1}-x_{i}\right| / x_{i+1}+x_{i}
$$

where, $x_{i}$ and $x_{i}+1$ are the values of two adjacent pixels along the slope of the profile and $L$ is the length of the slope. $\mathrm{L}$ was different in each case and was manually checked to avoid including regions on each side of the slope in the calculation.

As an optimal denoising approach is expected to reduce the noise by increasing SNR, without however introducing significant changes to the mean value within organs or tumors of interest, we finally measured the percentage of mean intensity bias (\%) in different ROIs placed inside the spheres/lesions and large homogeneous areas (such as the lungs and the liver for instance), defined as:

$$
\text { Intensity_bias }=\frac{\text { Mean_ROI }(I w)-M e a n_{-} R O I(I)}{M e a n_{-} R O I(I)} \times 100
$$

Where Mean_ROI(Iw) is the mean uptake value measured in the ROI on filtered image and Mean_ROI(I) is the corresponding mean uptake measured in the ROI reported on the original noisy image.

Significance of the difference between the various configurations and denoising methods was assessed using KruskalWallis tests, a non-parametric test appropriate for small, non Gaussian distributed samples. 


\section{RESULTS}

\subsection{Simulated images}

\subsubsection{Choice of the neighboring window size}

According to the results obtained using different window sizes in WD, CD and WCD applied to the IEC phantom dataset, although this choice had less important impact on WCD than on WD and CD, the optimal size was found to be $5 \times 5 \times 5$, with neither improvement nor decrease of performance with larger windows, as shown in figure 6 on 4 spheres of the IEC phantom chosen for illustration. Therefore the entire analysis from here onwards will be presented with results using a window size of 5 .
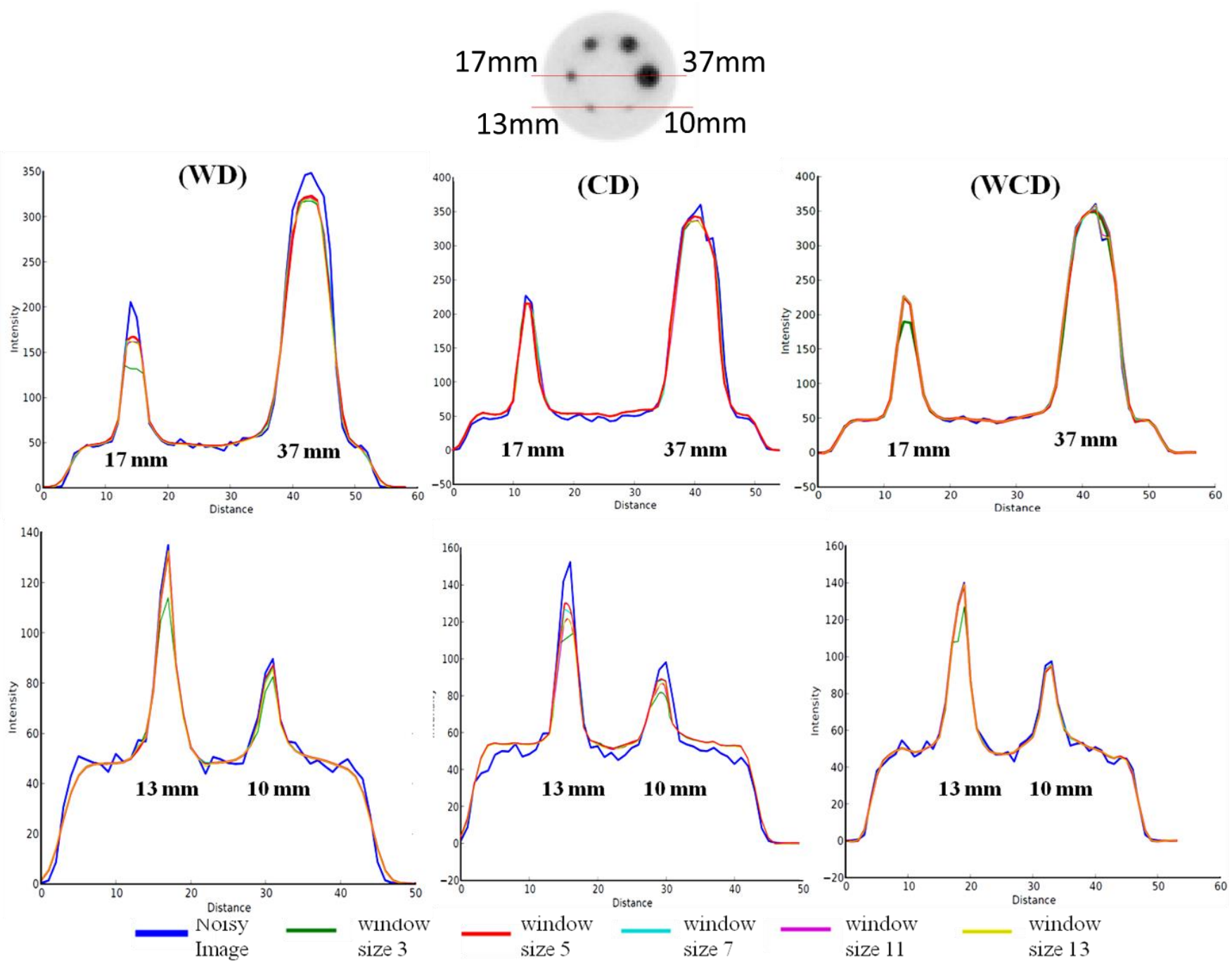

Figure 6: Comparison of profiles obtained on 4 different spheres of the IEC phantom considering the 3 different denoising schemes, and the 5 different sizes for the neighboring window, with respect to the original noisy image profile (blue).

\subsubsection{Homogeneous cylindrical phantom}

Results for the cylindrical phantom are illustrated in figures 7 and 8 (for the 20 million coincidences case) and provided fully in tables I and II for different count statistics. Qualitative results are presented in figure 7 before and after denoising by the three approaches with the respective residual images. It can be visually noticed that the edges of the cylinder are better preserved with CD and WCD compared to the WD which resulted in more blurred edges. Profiles going through the edges of the cylinder are illustrated in figure 8. 


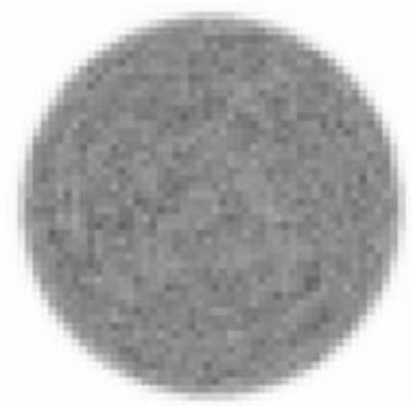

(a)

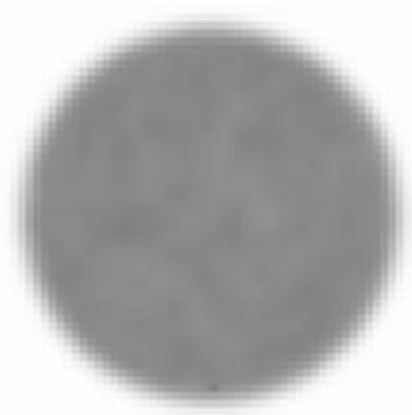

(b)

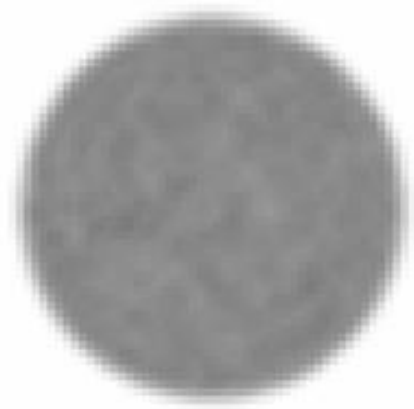

(c)

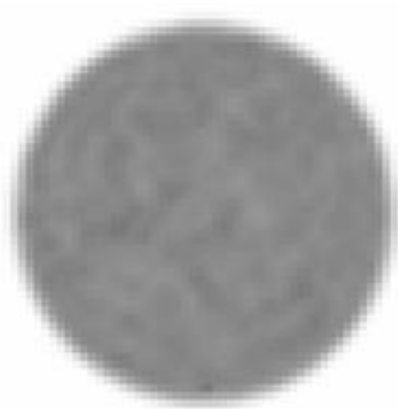

(d)

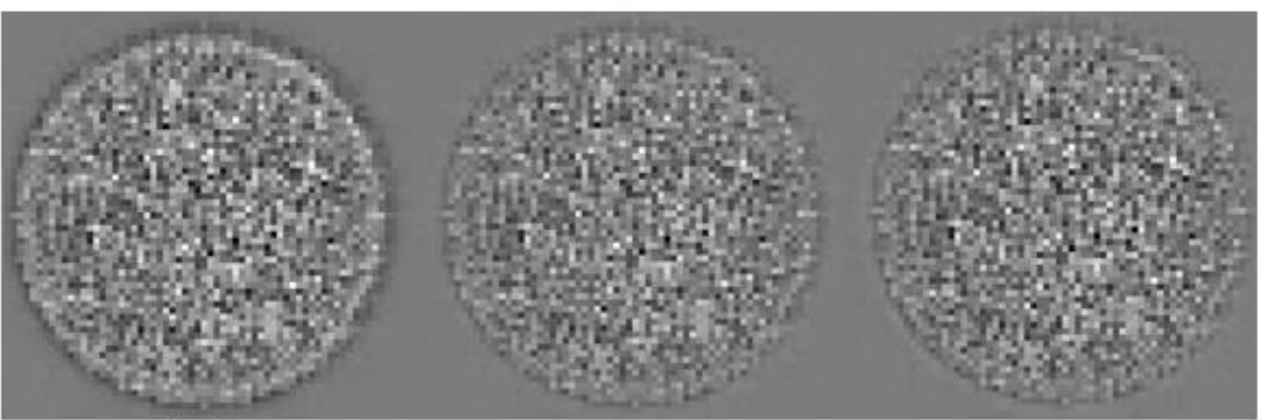

(e)

(f)

(g)

Figure 7: Cylindrical phantom illustration (20 million coincidences), before (a) and after (b-d) denoising by the three approaches: WD (b), CD (c) and WCD (d), along with their associated residual images (e-g).

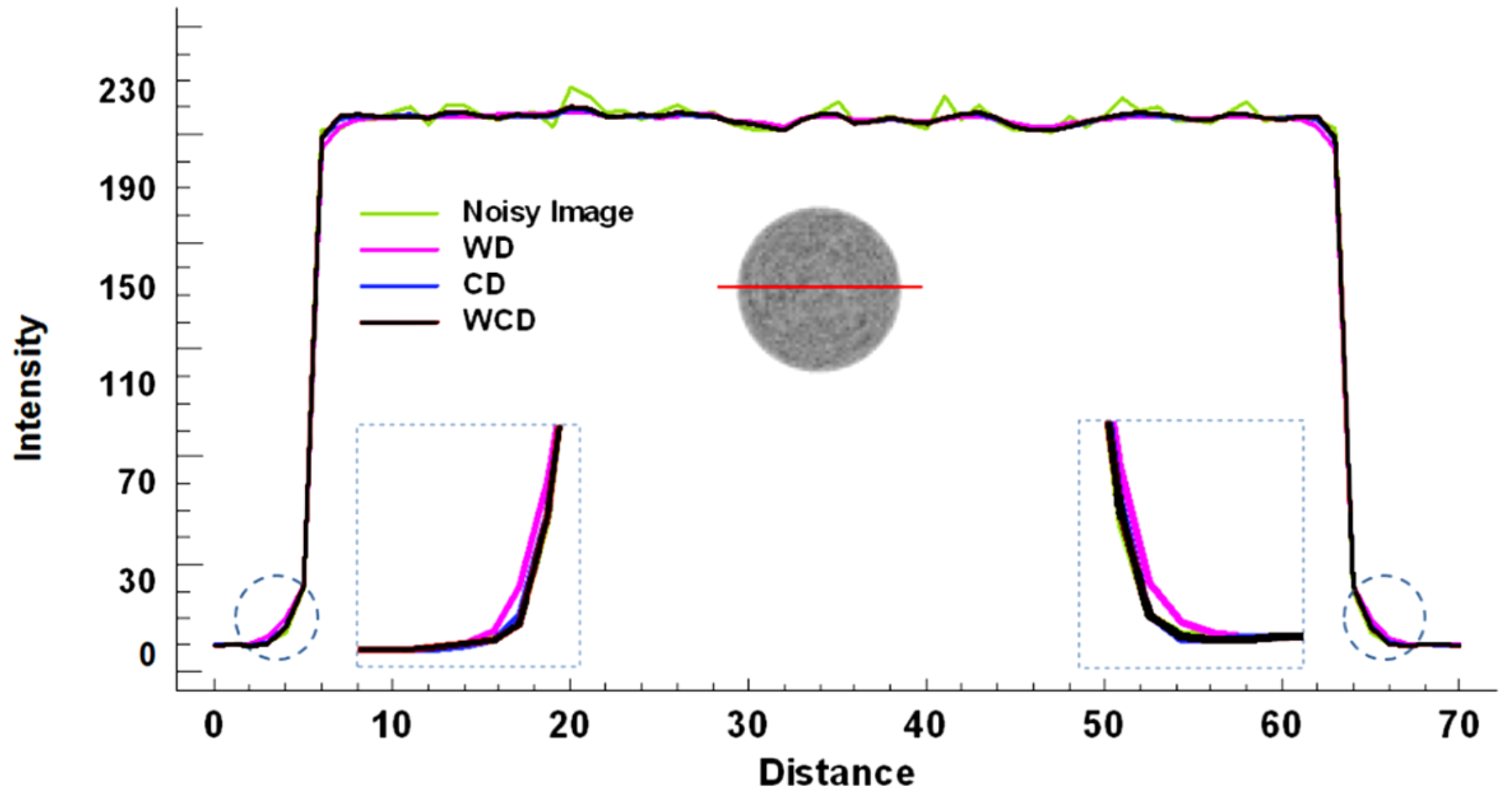

Figure 8: Profile plot for the cylindrical phantom simulated dataset (20 millions coincidences case). 


\begin{tabular}{cccccc}
\hline \hline $\begin{array}{c}\text { Number of } \\
\text { coincidences }\end{array}$ & $25 \mathrm{M}$ & $20 \mathrm{M}$ & $15 \mathrm{M}$ & $10 \mathrm{M}$ & $5 \mathrm{M}$ \\
\hline WD & 18.5 & 15.6 & 22.1 & 12.8 & 12.5 \\
CD & 4.6 & 2.7 & 5.4 & 2.1 & 2.9 \\
WCD & 0.8 & 0.3 & 3.2 & 0.6 & 1.1 \\
\hline \hline
\end{tabular}

Table 1: Mean local contrast decrease (\%)

WCD led to significantly lower mean local contrast decreases with $1.2 \pm 1.1 \%$ vs. $3.4 \pm 1.4 \%$ for CD and $16.3 \pm 4.1 \%$ for WD $(p=0.004)$ (table 1). No significant differences were found with respect to the number of coincidences $(p>0.7)$.

\begin{tabular}{cccccc}
\hline \hline $\begin{array}{c}\text { Number of } \\
\text { coincidences }\end{array}$ & $25 \mathrm{M}$ & $20 \mathrm{M}$ & $15 \mathrm{M}$ & $10 \mathrm{M}$ & $5 \mathrm{M}$ \\
\hline WD & 446.1 & 426.4 & 397.0 & 402.7 & 343.0 \\
CD & 223.2 & 212.4 & 198.7 & 197.8 & 174.0 \\
WCD & 218.1 & 208.2 & 195.0 & 195.2 & 170.2 \\
\hline \hline
\end{tabular}

Table 2: SNR increase (\%)

Significantly higher SNR increases across the five configurations (5M to $25 \mathrm{M}$ coincidences) were obtained with the WD method (mean \pm SD of $403 \pm 39 \%)$ vs. CD and WCD ( $p=0.008)$ (table 2$)$. CD and WCD still led to large improvements with a mean \pm SD of $201 \pm 18 \%$ and of $197 \pm 18 \%$ respectively, that were not significantly different $(p>0.5)$. Again, no significant differences were found with respect to the number of coincidences.

Regarding the intensity within a large homogeneous region in the cylinder, the mean intensity bias (mean \pm SD calculated across the entire range of configurations) was negligible for the three approaches with less than $0.15 \pm 0.1 \%$, $0.05 \pm 0.02 \%$ and $0.01 \pm 0.03 \%$ for $\mathrm{WD}, \mathrm{CD}$ and $\mathrm{WCD}$ respectively although statistically speaking WD results were significantly higher than $\mathrm{CD}$ and $\mathrm{WCD}(p<0.04)$.

\subsubsection{IEC phantom}

Results for the IEC phantom simulation are illustrated in figure 9 for the same configuration as in figure 5b. WCD led to almost no loss of resolution and structures, contrary to WD and CD approaches, as it can be noticed from the residual images and as the profiles plotted at the boundaries of all six spheres shown in figure 10 illustrate. The worst results were obtained with Gaussian filtering.

These results provide an estimation of the loss of local contrast induced by the different denoising processes on the objects of interest. The Gaussian filter led to an important loss of contrast for all spheres, especially for $28 \mathrm{~mm}$ and below. The wavelet approach led to a loss of intensity and contours blurring for the three biggest spheres (37, 28 and $22 \mathrm{~mm}$ ) but not for the smallest ones $(10,13$ and $17 \mathrm{~mm})$. On the contrary, the curvelet approach led to a loss of intensity and contours blurring for the 10 and $13 \mathrm{~mm}$ spheres but not for larger spheres. Finally, the profiles drawn on all the spheres in the WCD image were found to be almost exactly the same as the one drawn on the original noisy image. 


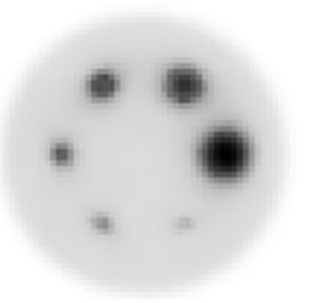

(b)

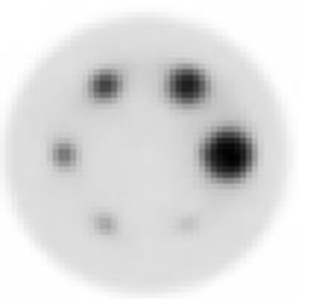

(c)

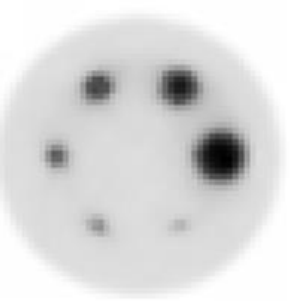

(d)

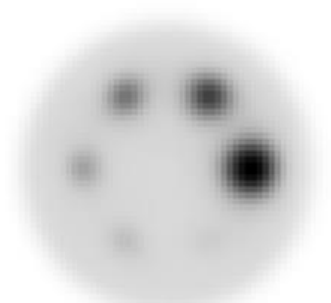

(e)

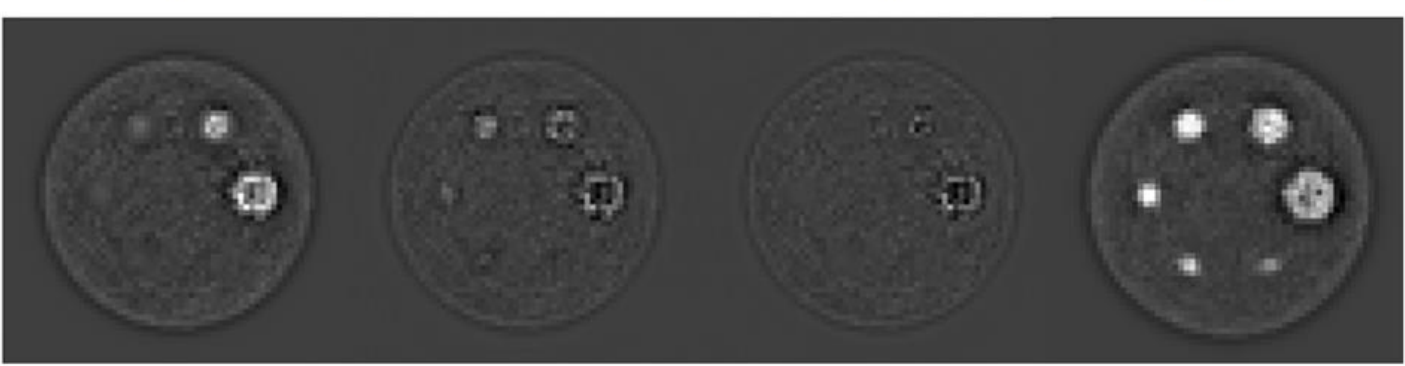

(f)

(g)

(h)

(i)

Figure 9: IEC phantom case with $4 \times 4 \times 4 \mathrm{~mm}^{3}, 60$ millions coincidences and 8:1 ratio, (a) before and (b-e) after denoising by (b) WD, (c) CD and (d) WCD and (e) Gaussian filter, along with their corresponding residual images (f-i).
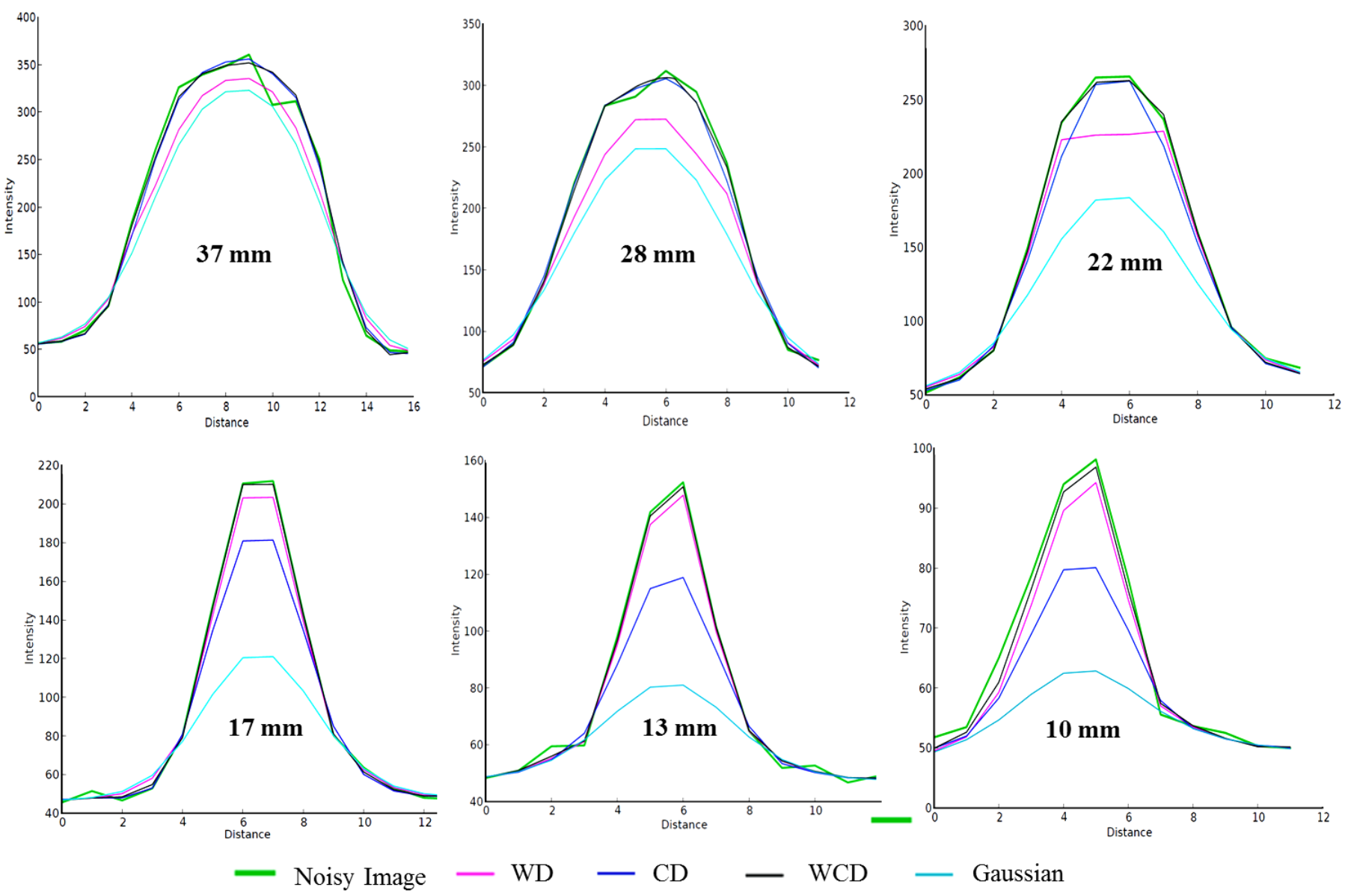

Figure 10: Profiles plots on all six spheres for the IEC phantom simulated dataset case with $4 \times 4 \times 4 \mathrm{~mm}^{3}, 60$ millions coincidences, $8: 1$ ratio. 


\begin{tabular}{ccccccc}
\hline \hline Sphere & $10 \mathrm{~mm}$ & $13 \mathrm{~mm}$ & $17 \mathrm{~mm}$ & $22 \mathrm{~mm}$ & $28 \mathrm{~mm}$ & $37 \mathrm{~mm}$ \\
\hline WD & $11.1 \pm 8.2$ & $14.5 \pm 14.0$ & $10.2 \pm 8.5$ & $16.9 \pm 10.2$ & $14.6 \pm 8.3$ & $12.9 \pm 11.1$ \\
CD & $13.3 \pm 13.6$ & $17.3 \pm 4.2$ & $7.0 \pm 5.0$ & $8.0 \pm 4.9$ & $5.4 \pm 10.0$ & $7.0 \pm 11.0$ \\
WCD & $0.2 \pm 6.7$ & $9.3 \pm 9.4$ & $3.7 \pm 4.6$ & $5.0 \pm 9.7$ & $5.3 \pm 9.4$ & $7.2 \pm 10.0$ \\
Gaussian & $63.3 \pm 8.5$ & $55.8 \pm 6.3$ & $42.1 \pm 7.3$ & $37.8 \pm 3.9$ & $17.1 \pm 3.5$ & $15.2 \pm 4.7$ \\
\hline \hline
\end{tabular}

Table 3: Mean \pm SD contrast decrease $(\%)$ calculated across the 12 configurations at the boundaries of the spheres

The mean contrast at the boundaries was calculated from the profiles illustrated in figure 10. WCD led to a significantly lower mean local contrast decrease across the entire range of configurations and spheres of $1.0 \pm 5.5 \%$ compared to $7.7 \pm 8.6 \%, 5.8 \pm 7.4 \%$ and $38.6 \pm 5.7 \%$ for $\mathrm{WD}, \mathrm{CD}$ and Gaussian $(p=0.01)$ (table 3 ). These results further confirmed that contour blurring was slightly higher for the largest ROIs using WD, and for the smallest ROIs using CD, although this trend was not significant $(\mathrm{p}=0.7)$.

\begin{tabular}{cccccccc}
\hline \hline ROI & $10 \mathrm{~mm}$ & $13 \mathrm{~mm}$ & $17 \mathrm{~mm}$ & $22 \mathrm{~mm}$ & $28 \mathrm{~mm}$ & $37 \mathrm{~mm}$ & Cylinder \\
\hline WD & $10.6 \pm 7.6$ & $21.6 \pm 3.6$ & $13.3 \pm 2.3$ & $13.9 \pm 5.7$ & $15.3 \pm 8.1$ & $13.5 \pm 0.2$ & $31.4 \pm 0.3$ \\
CD & $28.7 \pm 3.9$ & $18.6 \pm 0.6$ & $12.2 \pm 1.4$ & $12.5 \pm 3.4$ & $16.8 \pm 7.9$ & $16.4 \pm 0.6$ & $24.2 \pm 0.8$ \\
WCD & $8.7 \pm 7.4$ & $16.4 \pm 3.1$ & $10.0 \pm 1.6$ & $12.2 \pm 3.6$ & $17.1 \pm 7.8$ & $16.7 \pm 0.6$ & $22.3 \pm 0.7$ \\
Gaussian & $29.1 \pm 4.7$ & $22.5 \pm 2.8$ & $13.6 \pm 4.3$ & $14.1 \pm 4.3$ & $15.4 \pm 6.7$ & $15.2 \pm 0.3$ & $34.2 \pm 0.5$ \\
\hline \hline
\end{tabular}

Table 4: Mean \pm SD SNR increase (\%) across the 12 configurations

In terms of SNR percentage increase, all the methodologies increased SNR with no significant difference between the three approaches nor with respect to the size of the spheres $(p>0.1)$, except using Gaussian filter for the two smallest spheres (table 4). WCD, CD, WD and Gaussian led to $+22.3 \pm 0.7 \%,+24.2 \pm 0.8 \%,+31.3 \pm 0.3 \%$ and $+34.2 \pm 0.5 \%$ SNR increase respectively.

\begin{tabular}{cccccccc}
\hline \hline ROI & $10 \mathrm{~mm}$ & $13 \mathrm{~mm}$ & $17 \mathrm{~mm}$ & $22 \mathrm{~mm}$ & $28 \mathrm{~mm}$ & $37 \mathrm{~mm}$ & Cylinder \\
\hline WD & $-0.9 \pm 1.3$ & $-1.5 \pm 1.4$ & $-1.0 \pm 0.8$ & $-1.8 \pm 1.5$ & $-4.0 \pm 3.6$ & $-8.2 \pm 7.0$ & $-7.1 \pm 3.8$ \\
CD & $-3.1 \pm 5.0$ & $-7.9 \pm 2.2$ & $-6.9 \pm 3.4$ & $-4.1 \pm 1.6$ & $-4.7 \pm 2.4$ & $-5.0 \pm 3.3$ & $-6.4 \pm 4.0$ \\
WCD & $0.3 \pm 0.4$ & $1.2 \pm 1.5$ & $0.4 \pm 0.2$ & $0.1 \pm 0.1$ & $0.4 \pm 0.5$ & $0.9 \pm 3.4$ & $1.7 \pm 1.3$ \\
Gaussian & $-61.9 \pm 5.3$ & $-53.2 \pm 4.1$ & $-41.8 \pm 5.7$ & $-40.5 \pm 3.6$ & $-14.2 \pm 3.8$ & $-11.7 \pm 4.9$ & $-8.3 \pm 2.1$ \\
\hline \hline
\end{tabular}

Table 5: Mean \pm SD mean intensity bias (\%) across the 12 configurations

Regarding intensity bias, on the one hand, the lowest changes were observed for WCD with a maximum mean intensity bias of $+1.7 \pm 1.3 \%$ for the background ROI, whereas the average for WCD across 12 configurations and all ROIs was $+0.7 \pm 0.6 \%$ (table 5). On the other hand, significantly higher biases were associated with WD and CD approaches ( $p=0.0008$ ), with up to $-8.2 \pm 7.0 \%$ for the sphere of $37 \mathrm{~mm}$ with $\mathrm{WD}$, and $-7.9 \pm 2.2 \%$ for the sphere of $13 \mathrm{~mm}$ with $\mathrm{CD}$. The Gaussian filter was associated with the largest intensity biases systematically and significantly higher than most other denoising schemes for all spheres, especially for the 4 smallest ones (mean intensity biases from -40 to $-60 \%$ ). 


\subsubsection{Brain simulation phantom}

Results for the brain simulation dataset are illustrated in figure 11. Qualitative evaluation was carried out from the residual images and using profiles through different ROIs (tables 6-7). WD led to a significant loss of resolution compared to the two other methodologies, as demonstrated by the profiles (figure 12, black arrows) showing a loss of intensity and contrast introduced by all three denoising approaches. However, CD and WCD led to a lower impact.

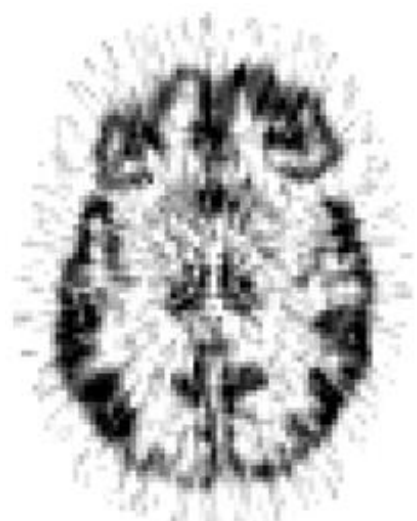

(a)

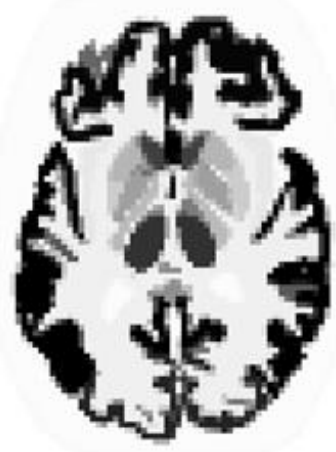

(b)

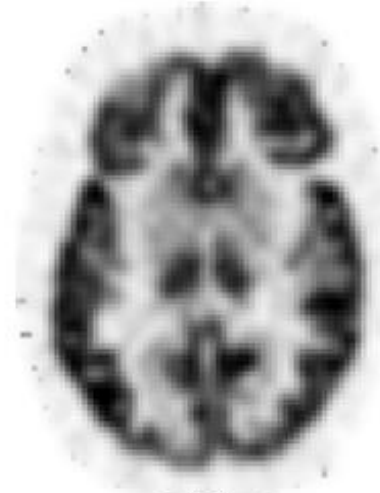

(c)

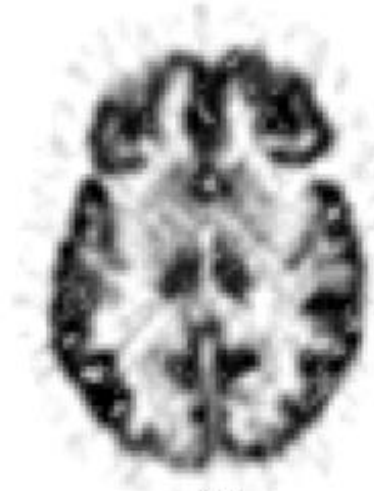

(d)

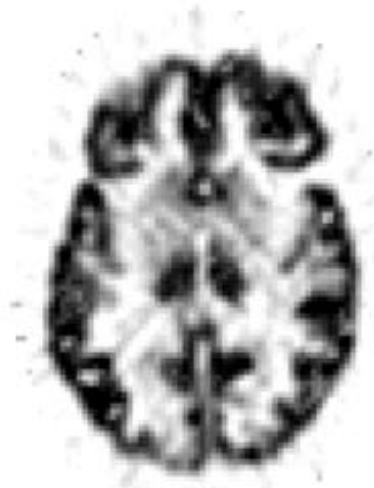

(e)

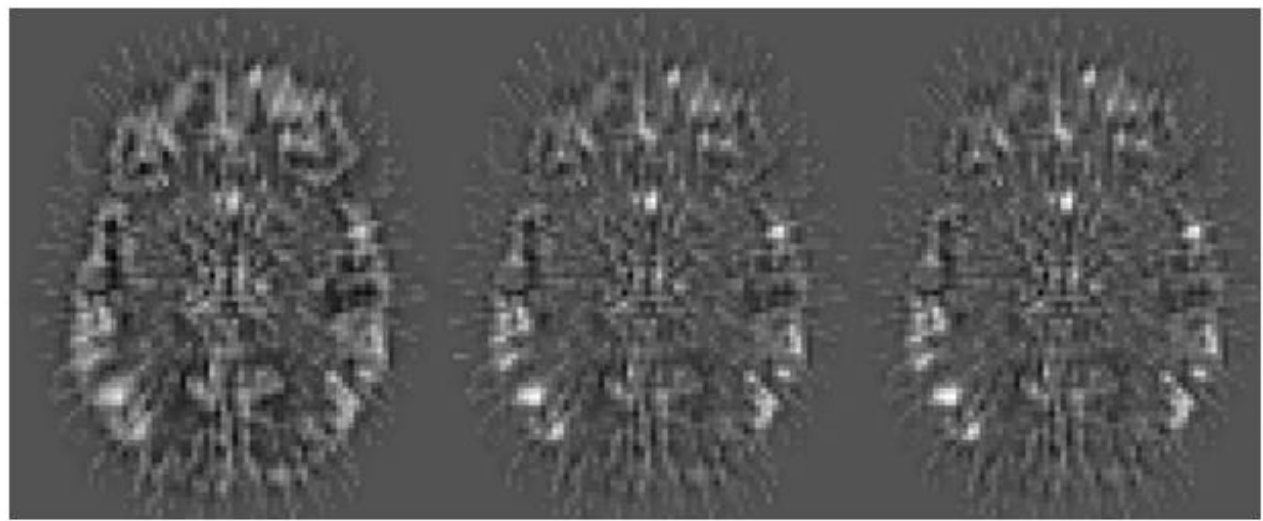

(f)

(g)

(h)

Figure 11: Brain simulation: PET image (a) and corresponding ground-truth (b). Results after denoising with WD (c), CD (d) and WCD (e), along their respective residual images (f-h). 


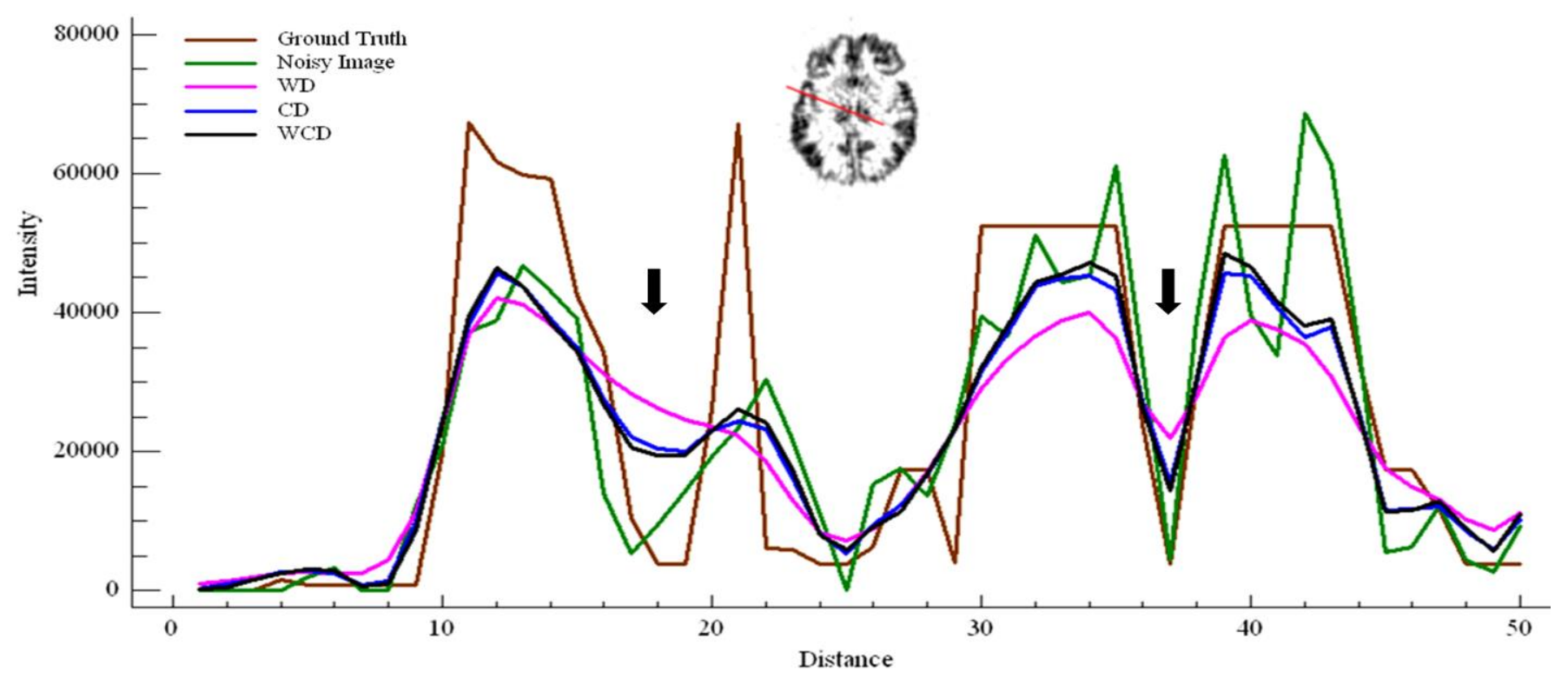

Figure 12: Profile plot on the simulated brain phantom acquisition

\begin{tabular}{cccc}
\hline \hline ROI & WD & CD & WCD \\
\hline Cerebellum & -1.6 & 0.5 & 0.5 \\
Putamen & -0.9 & 1.4 & 1.5 \\
Lat Front Gyrus & -2.5 & 2.4 & 2.3 \\
Amygdale & -2.4 & 2.2 & 2.0 \\
Hippocampus & -11.6 & -4.0 & -2.4 \\
Caudate & -14.1 & -2.5 & -0.4 \\
Thalamus & -21.9 & -15.0 & -14.0 \\
Sup Occ Gyrus & -15.2 & -4.8 & -2.7 \\
Med Front Gyrus & -22.2 & -14.7 & -13.4 \\
Sup Front Gyrus & -24.2 & -14.8 & -12.9 \\
\hline \hline
\end{tabular}

Table 6: mean local contrast decrease (\%)

This qualitative evaluation was confirmed by the measurements of the contrast at the boundaries of ten different structures such as for example the putamen, the amygdale or the frontal gyrus (table 6). The loss of resolution measured by the mean local contrast decrease calculated across all these profiles was of $12.6 \pm 6.7 \%, 17.5 \pm 16.2 \%$ and $38.3 \pm 27.9 \%$ for WCD, $\mathrm{CD}$ and WD respectively. WD and CD methods also led to a slightly higher intensity bias compared to the noisy image for all the ROIs with a mean decrease of respectively $-11.7 \pm 9.3 \%$ and $-4.9 \pm 7.3 \%$ compared to $-3.9 \pm 6.7 \%$ for WCD. This trend was however not significant $(p=0.08$ ), except for the 6 last ROIs (from hippocampal to the Sup Front Gyrus) for which WCD and CD led to a significantly lower bias than WD $(p=0.02)$. 


\begin{tabular}{cccc}
\hline \hline ROI & WD & CD & WCD \\
\hline Cerebellum & 20.3 & 15.1 & 14.2 \\
Putamen & 39.2 & 15.7 & 15.4 \\
Lat Front Gyrus & 23.0 & 13.2 & 11.8 \\
Amygdale & 29.9 & 17.0 & 17.3 \\
Hippocampus & 23.6 & 11.3 & 9.7 \\
Caudate & 12.1 & 7.2 & 5.8 \\
Thalamus & 41.6 & 19.6 & 18.4 \\
Sup Occ Gyrus & 15.3 & 8.4 & 7.1 \\
Med Front Gyrus & 14.8 & 5.7 & 2.8 \\
Sup Front Gyrus & 15.2 & 7.9 & 6.5 \\
\hline \hline
\end{tabular}

Table 7: SNR increase (\%)

WD led to significantly higher SNR increases $(p=0.006)$ over all the ROIs (table 7$)$ of $+23.5 \pm 10.4 \%$, compared to $+12.1 \pm 4.7 \%$ and $+10.9 \pm 5.3 \%$ for $\mathrm{CD}$ and $\mathrm{WCD}$ (no significant difference) respectively.

\subsection{Clinical images}

\subsubsection{First dataset (large voxels, post reconstruction smoothing)}

Qualitatively, the images of the first dataset were efficiently denoised whatever method was used (see figure 13), although the plot profile (figure 14) going through the whole body and an identified tumor illustrates the fact that WD led to a loss of intensity and contrast (with a blurring of the organs edges), contrary to the results of the CD and WCD methodologies.

As it can be seen on the residual images of figure 13, WD led to a visible general loss of resolution, more pronounced at the edges, whereas the tumor region appeared unmodified in comparison. CD induced a lower loss of details at the edges and for all the anisotropic structures within the image, although it introduced a visible change in and around the tumor, which can be considered as a quasi-isotropic structure due to its size. On the other hand, the WCD approach was associated with the minimum changes in both tumor and organ edges.

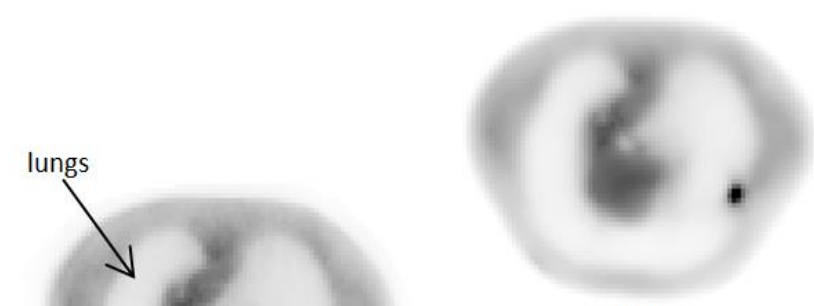

(b)

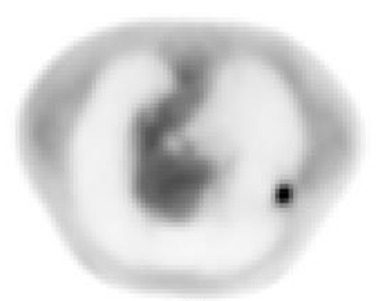

(c)

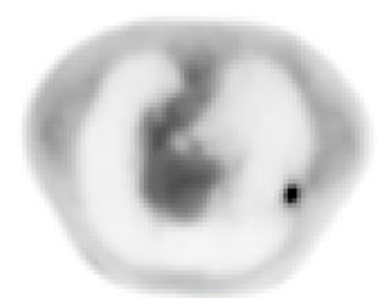

(d)
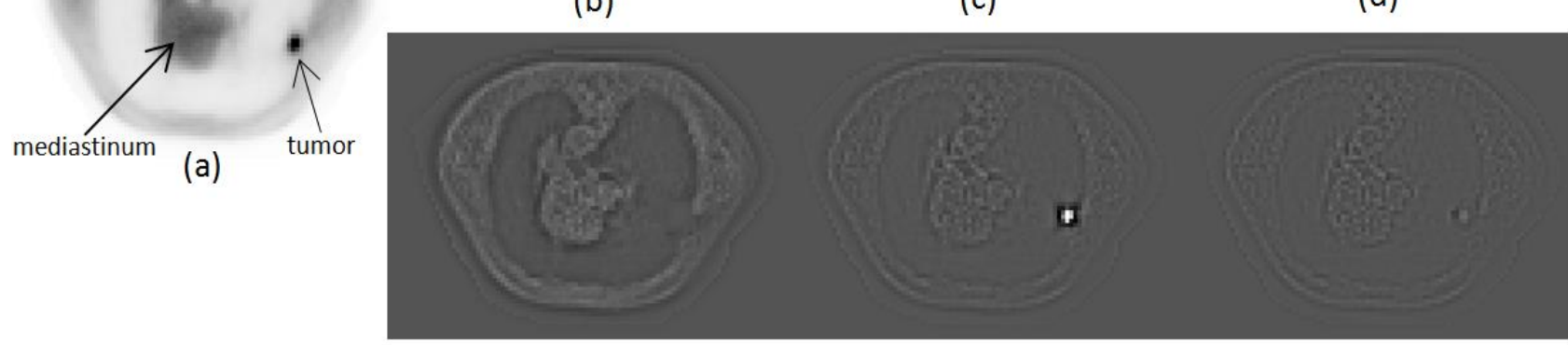

(e)

(f)

(g)

Figure 13: One of the first clinical dataset PET image (a) before and (b-d) after denoising with (b) WD, (c) CD and (d) WCD, along with their associated residual images $(\mathrm{e}-\mathrm{g})$. 


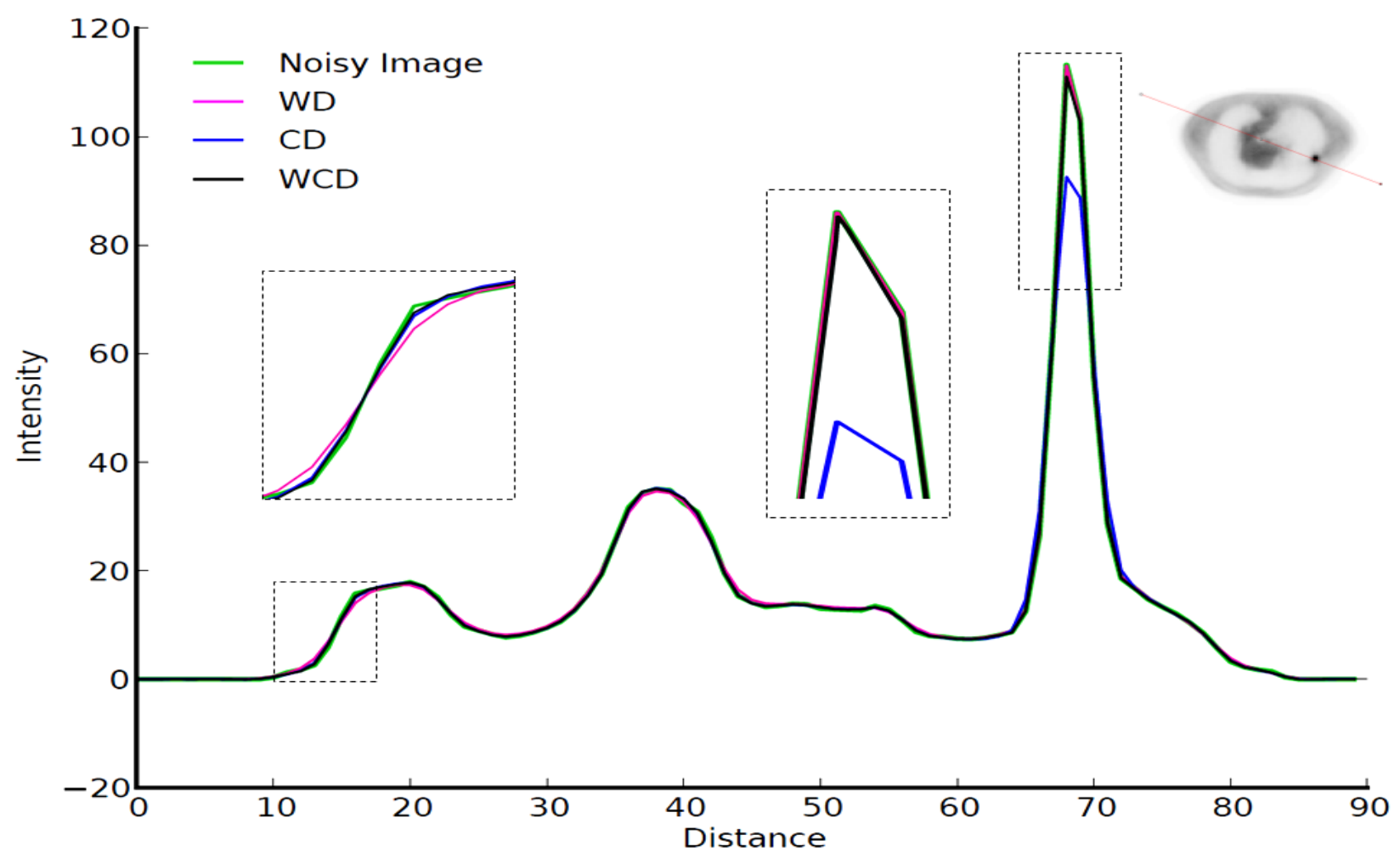

Figure 14: Profile plot on the whole body PET image from first clinical dataset illustrated in figure 5.

\begin{tabular}{cccc}
\hline \hline ROI & WD & CD & WCD \\
\hline Tumor & $7.1 \mathrm{e}^{-3} \pm 3.4 \mathrm{e}^{-3}$ & $-0.2 \pm 0.4$ & $1.3 \mathrm{e}^{-2} \pm 0.1$ \\
Lung & $-4.2 \mathrm{e}^{-2} \pm-0.1$ & $-0.3 \pm 1.0$ & $4.2 \mathrm{e}^{-2} \pm 0.1$ \\
Mediastinum & $-0.2 \pm-0.2$ & $1.2 \pm 2.5$ & $0.2 \pm 0.4$ \\
Liver & $-7.1 \pm-7.7$ & $-5.2 \pm 2.1$ & $0.9 \pm 0.5$ \\
\hline \hline
\end{tabular}

Table 8: Mean \pm SD intensity bias $(\%)$ for clinical dataset 1

\begin{tabular}{cccc}
\hline \hline ROI & WD & CD & WCD \\
\hline Tumor & $4.1 \pm 1.1$ & $7.4 \pm 2.6$ & $2.6 \pm 2.2$ \\
Lung & $5.7 \pm 5.2$ & $2.0 \pm 1.4$ & $2.0 \pm 0.6$ \\
Mediastinum & $7.8 \pm 2.3$ & $4.9 \pm 1.4$ & $3.6 \pm 1.0$ \\
Liver & $10.7 \pm 3.7$ & $4.7 \pm 0.8$ & $3.5 \pm 1.3$ \\
\hline \hline
\end{tabular}

Table 9: Mean \pm SD contrast decrease $(\%)$ for clinical dataset 1

This was further confirmed by the mean intensity bias (table 8) and by the local contrast decrease (table 9) obtained by the different approaches in the tumor, lungs, mediastinum and liver. WCD better preserved both the local contrast and intensity of the noisy image with a maximum local contrast decrease of 3.6\% (table 9) and a maximum intensity bias of $0.9 \%$ (table 8 ), contrary to WD and CD that led to $10.7 \%$ and $7.4 \%$ local contrast loss and $7.1 \%$ and $5.1 \%$ intensity bias for WD and CD respectively. This trend of lower bias associated with WCD was however not significant, except for the liver for which WCD led to a significantly lower bias $(p=0.002)$. On the other hand, contrast decreases associated with WCD were significantly lower than for WD and CD $(p<0.05)$ (table 9). 


\begin{tabular}{cccc}
\hline \hline ROI & WD & CD & WCD \\
\hline Tumor & $8.2 \pm 5.3$ & $11.2 \pm 7.6$ & $3.2 \pm 1.9$ \\
Lung & $16.4 \pm 6.6$ & $8.2 \pm 3.9$ & $5.9 \pm 2.8$ \\
Mediastinum & $13.6 \pm 7.8$ & $7.3 \pm 3.9$ & $5.2 \pm 3.0$ \\
Liver & $13.7 \pm 5.7$ & $6.2 \pm 3.0$ & $4.4 \pm 2.3$ \\
\hline \hline
\end{tabular}

Table 10: Mean \pm SD SNR increase (\%) for clinical dataset 1

Significantly higher SNR improvements in large homogeneous regions (lung, liver) (table 10) were obtained with WD over CD and WCD ( $p=0.01)$. The highest mean SNR increase $(+16.4 \pm 6.6 \%)$ was obtained for the lungs with WD and the lowest $(+3.2 \pm 1.9 \%)$ for the tumor with WCD.

\subsubsection{Second dataset (small voxels, no post reconstruction smoothing)}

Qualitatively, the images of the second clinical dataset were also efficiently denoised whatever method used (figure 15). The plot profile (figure 16) going through the whole body and an identified lesion, however, illustrates the fact that WD led to a higher loss of intensity and contrast (with a blurring of the organs edges), compared to the results obtained with the CD and WCD methodologies. According to the residual images (figure 15), WD led to a visible general loss of resolution, more pronounced in the area of the lesion exhibiting a complex anisotropic shape. In this illustrated case, CD induced a lower loss of details both at the edges and for the tumor structure; and the WCD approach was associated with the minimum modification of both tumor and overall organ edges.

(b)

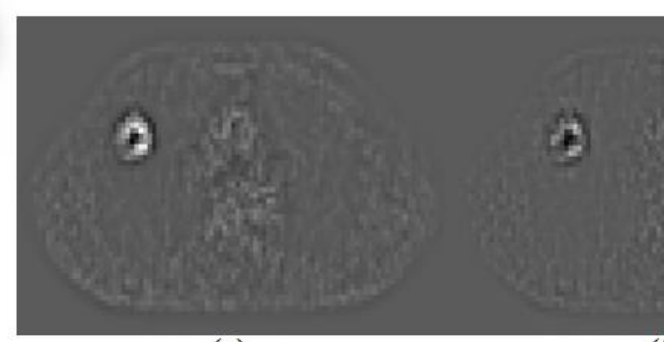

(e)

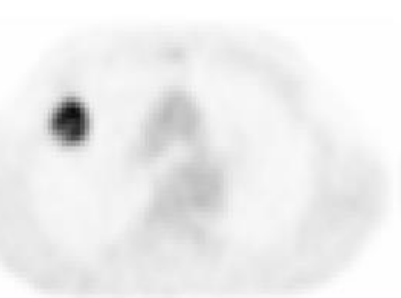

(c)

(d)

(a)

(f)

(g)

Figure 15: PET image of figure 5e before (a) and after denoising with WD (b), CD (c) and WCD (d), with their associated residual images (e-g). 


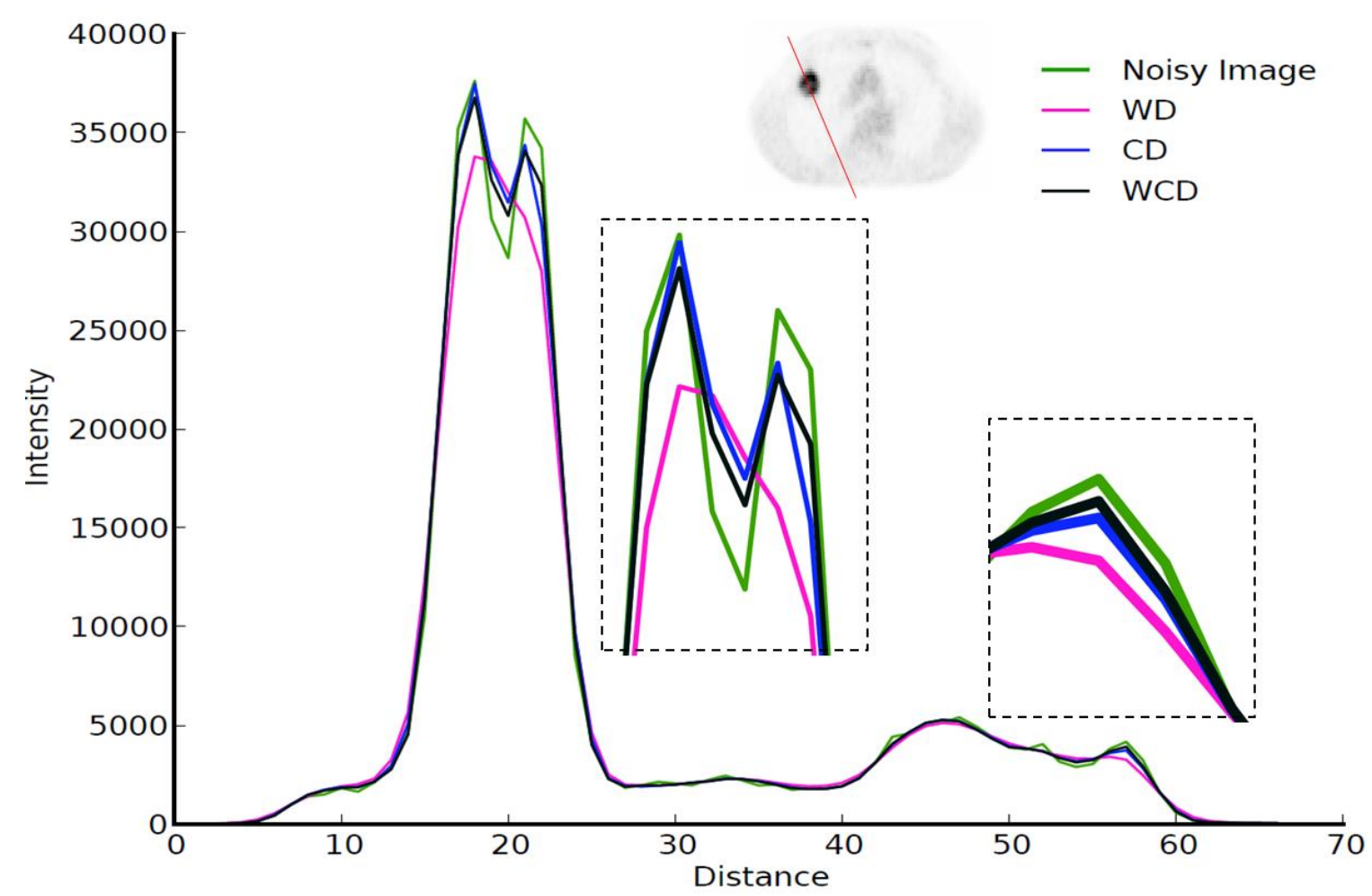

Figure 16: Profile plot on the whole body PET image from second clinical dataset illustrated in figure 5e.

\begin{tabular}{cccc}
\hline \hline ROI & WD & CD & WCD \\
\hline Tumor & $-7.5 \pm 4.2$ & $-1.9 \pm 1.3$ & $-1.6 \pm 0.9$ \\
Lung & $-2.1 \pm 1.8$ & $-0.2 \pm 0.9$ & $-4.9 \mathrm{e}^{-2} \pm 0.2$ \\
Mediastinum & $-1.9 \pm 0.7$ & $-0.2 \pm 1.1$ & $-4.7 \mathrm{e}^{-2} \pm 0.3$ \\
Liver & $-1.6 \pm 0.8$ & $-0.3 \pm 1.7$ & $-0.1 \pm 0.2$ \\
\hline \hline
\end{tabular}

Table 11: Mean \pm SD intensity bias (\%) for clinical dataset 2

This was further confirmed by the mean intensity bias (table 11) obtained by the different approaches. CD and WCD better preserved the intensity of the tumor with a significantly lower bias of respectively $-1.9 \%$ and $-1.6 \%$, compared to -7.5 for the WD $(p<0.05)$. Bias was also significantly lower for WCD and CD vs. WD for the other ROIs (table 11).

\begin{tabular}{cccc}
\hline \hline ROI & WD & CD & WCD \\
\hline Tumor & $11.2 \pm 4.4$ & $5.7 \pm 2.3$ & $4.3 \pm 2.6$ \\
Lung & $7.6 \pm 6.1$ & $3.2 \pm 2.4$ & $3.1 \pm 1.5$ \\
Mediastinum & $8.2 \pm 2.4$ & $4.1 \pm 1.1$ & $3.6 \pm 1.7$ \\
Liver & $8.4 \pm 4.5$ & $3.8 \pm 1.9$ & $3.7 \pm 1.7$ \\
\hline \hline
\end{tabular}

Table 12: Mean \pm SD contrast decrease (\%) for clinical dataset 2

WCD also better preserved the local contrast of the tumor with a significantly lower maximum decrease of $4.3 \%$ compared to $11.2 \%$ and $5.7 \%$ for the WD and CD respectively ( $p=0.02)$ (table 12 ). Difference with WD was also significant for the other ROIs. 


\begin{tabular}{cccc}
\hline \hline ROI & WD & CD & WCD \\
\hline Tumor & $-1.2 \pm 4.7$ & $4.4 \pm 5.8$ & $3.6 \pm 3.9$ \\
Lung & $16.0 \pm 5.4$ & $12.5 \pm 6.4$ & $13.0 \pm 6.8$ \\
Mediastinum & $22.3 \pm 6.4$ & $16.4 \pm 5.7$ & $16.7 \pm 7.1$ \\
Liver & $29.4 \pm 4.9$ & $20.4 \pm 6.9$ & $19.9 \pm 7.8$ \\
\hline \hline
\end{tabular}

Table 13: Mean \pm SD SNR increase $(\%)$ for clinical dataset 2

Contrary to the first clinical dataset, SNR improvements were not significantly different between the three denoising approaches $(p=0.8)$ and absolute values were also higher with respect to the first clinical dataset (table 13).

\section{DISCUSSION}

In this paper we compared three different denoising approaches, based on wavelets, curvelets and a combination of both. The aim of this work was to develop a denoising method dedicated to PET images that would provide efficient denoising while preserving as much as possible the original quantitative and structural (such as shapes and spatial features) information of structures of interest in the image. This is especially important by considering denoising as a first pre-processing step occurring before other image processing and analysis steps, such as partial volume effects correction, segmentation, and uptake heterogeneity characterization which is an important trend in the PET imaging community today, especially for therapy response studies (Chua et al. 2011).

The results we obtained on the simulated IEC phantom by applying a post-reconstruction Gaussian filter with a FWHM of $5 \mathrm{~mm}$, corresponding to clinical systems standard, demonstrated the major intensity biases and contrast loss associated with this approach. Contrary to the Gaussian filter, the denoising in the wavelet domain is considered as a state-of-the-art technique for PET imaging. It allows modifying voxel values at different levels of resolution, making possible in most instances the distinction between contrast at boundaries and noise. However, it suffers from some limitations due to its non optimal processing of edge discontinuities. A new multi scale geometric approach, the CT, allows extending the wavelet properties to account for directional properties in the image, such as edges. However, WT stays optimal for isotropic structures. In order to address the issue of resolution loss and mean intensity bias (observed in the presented results) associated with such standard denoising, we consequently considered a strategy combining WT and CT.

The initial noisy image is first denoised by a wavelet approach. Different wavelet transform and wavelet denoising techniques can be used at this step, such as, for instance, the standard SureShrink approach (Donoho and Johnstone 1995 ) purposely devised for PET (Turkheimer et al. 1999). We however decided to use a more recent wavelet denoising algorithm, namely the local adaptative BiShrink filter (Sendur and Selesnick 2002) that outperforms the SureShrink approach as it takes into account the statistical relationships between wavelet coefficients. Once the initial noisy image is processed, the discarded edges information are then extracted from the residual image via a local approach of the multi scale curvelet analysis (Starck et al. 2002). The final image is then a combination of the wavelet denoising result and the edges information recovered through the curvelet analysis. 
The respective advantages/drawbacks of WD and CD were well illustrated when applied to the simulated IEC phantom dataset containing homogeneous spheres of varying diameters. Whereas improvements were higher for WD over CD regarding the two smallest spheres that can be considered as isotropic structures, $\mathrm{CD}$ led to better performances on the largest spheres. Our proposed scheme led to overall better performances than CD or WD alone, demonstrating it did manage to benefit from the best of both approaches.

On the one hand, the results we obtained overall on both simulated and clinical datasets, representative of what can be obtained with current state-of-the-art PET scanners, suggest that WD generates the highest SNR increases, although for some cases the differences are not significant. On the other hand, the mean intensity bias and loss of contrast associated with WD might compromise the quantitative accuracy of the resulting denoised images. From a clinical and quantitative point of view, the optimal denoising method should be the one that best preserves the original structural, geometric and quantitative information, while still improving SNR. This is especially true in the current context of exploiting further the information contained in PET images beyond the standard $\mathrm{SUV}_{\max }$ by considering automated characterization method such as spatial features analysis (Tixier et al. 2012). The proposed methodology combining WD and $\mathrm{CD}$ techniques corresponds to that requirement by exploiting both individual transforms. It provided satisfactory results in terms of SNR increase on all datasets. It was also associated with more limited resolution loss than the two other techniques, although the magnitude of improvement was dependent on the considered dataset and not significant for all the tested images.

The first comment regarding our methodology concerns the lower SNR results for WCD compared to WD or CD only. This difference can be primarily explained because of the preservation of the mean intensity values associated with the proposed approach relative to the WD and CD methods, with a direct impact on the ratio in equation 12 . The general performances of the denoising approaches were also different between the various datasets. Up to more than $200 \%$ of SNR increase was obtained for the homogeneous cylinder, whereas less striking increases were obtained for the IEC simulated phantom (around 10 to 30\%), and even less important values for the brain simulated phantom and the clinical images (with SNR gain from 3\% up to 30\% depending on the acquisition and reconstruction parameters). This can first be explained by the differences in the image reconstruction process. It was performed without post-filtering in the case of the simulated datasets, similarly to the second clinical dataset for which the highest SNR gains were obtained, and contrary to the first clinical dataset generated using a post-reconstruction Gaussian filter with a FWHM of 5mm, for which the gain was much lower. This can be directly related to the results observed using Gaussian filter on the IEC simulated phantom that led to images with improved SNRs but also major loss of structures and contrast, as well as intensity biases. This further stresses the potential benefit from applying denoising techniques such as the ones proposed in the present work on clinical images reconstructed without post-reconstruction smoothing. The results we obtained on the clinical datasets with incorporated post-reconstruction filtering demonstrated that since the images had already been smoothed, an additional denoising did not improve the SNR much further. A second element of explanation is the complexity and sizes of structures. In the homogeneous cylinder for which SNR gains of more than 
$200 \%$ were obtained, there was no structure and only an homogeneous background, which is the most simple case. In the simulated IEC phantom (for which SNR gains of 10-30\% were obtained), the structures are very simple, consisting of an homogeneous background with simple spheres containing homogeneous uptake. This is obviously a simplification of real clinical images that contain a much broader range of shapes, sizes, and uptake heterogeneities. The range of SNR improvement values obtained on the brain phantom dataset were actually much closer to the results obtained on the clinical dataset, which strengthens this argument. A third and final element is the the noise estimation (equation 3) as the proposed WCD approach is directly dependent on the performance of the first step (WD). If this approach led to good results on phantom considering a stationary noise and a local area (cylinder, brain), for wholebody PET images, the noise estimation may be less robust due to the non stationary nature of the noise within the field of view. The noise estimation from the variance measurement at the first sub-band of the WT may be non optimal and underestimate the variance in other sub-bands. This represents a limitation not only for our approach but also for all WD or $\mathrm{CD}$ techniques previously published and should be considered in future studies to improve the general performance of PET denoising processes.

A second comment concerns the choice of applying WD and CD sequentially. As the "à trous" WT is not orthogonal, performing CD before WD in the WCD process would theoretically lead to different results. However, the aim of this study was to propose an improved wavelet-based denoising (WD being considered as the current state-of-the-art method) by analyzing its residual image and recovering the structures lost during the initial denoising process. Furthermore, starting with $\mathrm{CD}$ would lead to a residual image containing noise and lost isotropic structures which may be harder to detect within the noisy residual image than anisotropic elements. We therefore chose to perform WD before $\mathrm{CD}$. From a theoretical point of view, most of the relevant edge information retained in the residual image after WD should be extracted by CD, even when these edges are lost amidst amplified noise (Starck et al. 2002). However, such an approach may still be suboptimal compared to a parallel analysis of the initial noisy image via WD and CD performed simultaneously and subsequently combined (Starck 2001). A full comparison with such an approach will however require a different implementation and a thorough comparison on the same datasets, which is out of the scope of the present study and will be considered for future work.

Although we investigated the performance of the method on a large spectrum of both simulated and clinical datasets, only a limited number of reconstruction algorithms and types of images could be considered in this first work. Future studies will consequently further validate the ability of our straightforward PET denoising method to achieve similar performance on PET images obtained using different reconstruction algorithms and scanners. For instance, the comparison between post-reconstruction denoising (such as the scheme proposed here) and improved image reconstruction algorithms, such as the incorporation of additional regularization (for example anatomical smoothing priors) or FBP with Hann filter, will be investigated in future studies. Improved noise estimation and alternative strategies combining WD and CD in a parallel way will also be investigated. 


\section{CONCLUSION}

In this paper, a denoising methodology for PET was presented, overcoming limitations encountered in the wavelet approaches currently considered as the state-of-the-art denoising filters for PET imaging. Our approach is based on the combination of a wavelet transform and a new multi resolution technique, namely the curvelet transform. These are combined in a new sequential filtering scheme. On all simulated and clinical datasets considered, the combined approach provided the best compromise between signal to noise ratio increase and preservation of both mean intensity and local contrast, independently of the sizes and shapes of the regions of interest. This new PET denoising approach does not require other imaging modality or complex parameter estimation to perform efficient denoising. Although the SNR increases were higher with wavelet- or curvelet-only approaches, these approaches were also associated with higher bias in the mean activity measured in different ROIs, as well as substantial local contrast and resolution loss. The wavelet-curvelet denoising might be a promising tool to replace simple filters such as Gaussian that are currently offered in state-of-the-art clinical PET systems to provide smoothed images.

Acknowledgements: We would like to thank Dr C Tsoumpas for providing the simulated brain dataset. 


\section{REFERENCES}

Alessio, A. and P. Kinahan (2006). PET image reconstruction. Nuclear Medicine. H. e. a. Elsevier. Philadelphia. 1.

Alpert, N. M., A. Reilhac, et al. (2006). "Optimization of dynamic measurement of receptor kinetics by wavelet denoising." Neuroimage 30(2): 444-451.

Boussion, N., C. Cheze Le Rest, et al. (2009). "Incorporation of wavelet-based denoising in iterative deconvolution for partial volume correction in whole-body PET imaging." Eur J Nucl Med Mol Imaging 36(7): 1064-1075.

Candes, E. J. (2006). "Fast discrete curvelet transforms." Multiscale Model. Simul. 5: 861-899.

Candès, E. J. and D. L. Donoho (2002). "New Tight Frames of Curvelets and Optimal Representations of Objects with Smooth Singularities." Stanford University.

Cands, E. (1998). Ridgelets: Theory and Applications, Stanford University: 1-125.

Chang, S. G., B. Yu, et al. (2000). "Adaptive wavelet thresholding for image denoising and compression." IEEE Trans Image Process 9(9): 1532-1546.

Chen, S. S. (2001). "Atomic decomposition by basis pursuit." SIAM REVIEW 43: 129-159.

Chua, S., J. Dickson, et al. (2011). "PET imaging for prediction of response to therapy and outcome in oesophageal carcinoma." Eur J Nucl Med Mol Imaging.

Crouse, M. S., R. D. Nowak, et al. (1998). "Wavelet-based statistical signal processing using hidden Markov models." $\underline{\text { Signal }}$ Processing, IEEE Transactions on 46(4): 886-902.

Donoho, D. L. (1995). "De-noising by soft-thresholding." Information Theory, IEEE Transactions on 41(3): $613-627$.

Donoho, D. L. and I. M. Johnstone (1994). "Ideal Spatial Adaptation by Wavelet Shrinkage." Biometrika 81(3): 425-455.

Donoho, D. L. and I. M. Johnstone (1995). "Adapting to Unknown Smoothness via Wavelet Shrinkage." Journal of the American Statistical Association 90(432): 1200-1224.

Dutilleux, P. (1987). An implementation of the 'algorithme a trous' to compute the wavelet transform'. Time-Frequency Methods and Phase Space. Springer-Verlag. 1: 298-304.

Easley, G., D. Labate, et al. (2008). "Sparse directional image representations using the discrete shearlet transform." Applied and Computational Harmonic Analysis 25(1): 25-46.

Hatt, M., C. Cheze le Rest, et al. (2009). "A fuzzy locally adaptive Bayesian segmentation approach for volume determination in PET." IEEE Trans Med Imaging 28(6): 881-893.

Helbert, D., P. Carre, et al. (2006). "3-D discrete analytical ridgelet transform." IEEE Trans Image Process 15(12): $3701-3714$.

Huo, X. (1999). Sparse Image Representation via Combined Transforms. Department of statistics. Standford Standford University.

Hyder, S. A. and R. Sukanesh (2011). "An efficient algorithm for denoising MR and CT images using digital curvelet transform." Adv Exp Med Biol 696: 471-480.

Jordan, K. (1990). "IEC emission phantom appendix performance evaluation of positron emission tomographs." Med. Public Health Res. Programme Eur. Commun.

Lamare, F., A. Turzo, et al. (2006). "Validation of a Monte Carlo simulation of the Philips Allegro/GEMINI PET systems using GATE." Physics in Medicine and Biology 51(4): 943-962.

Lamare, F., A. Turzo, et al. (2006). "Validation of a Monte Carlo simulation of the Philips Allegro/GEMINI PET systems using GATE." Phys Med Biol 51(4): 943-962.

Le Maitre, A., W. P. Segars, et al. (2009). Incorporating Patient-Specific Variability in the Simulation of Realistic Whole-Body 18F-FDG Distributions for Oncology Applications. Proceedings of the IEEE.

Le Pogam, A., M. Hatt, et al. (2011). "Evaluation of a 3D local multiresolution algorithm for the correction of partial volume effects in positron emission tomography." Med Phys 38(9): 4920-4923.

Lodge, M. A., A. Rahmim, et al. (2010). "Simultaneous measurement of noise and spatial resolution in PET phantom images." Phys Med Biol 55(4): 1069-1081.

Mallat, S. G. (1989). "A theory for multiresolution signal decomposition: the wavelet representation." Pattern Analysis and Machine Intelligence, IEEE Transactions on 11(7): 674-693.

Mallat, S. G. (2009). A wavelet tour of signal processing : the sparse way. Amsterdam ; Boston, Elsevier/Academic Press.

Reader, A. J., S. Ally, et al. (2002). "One-pass list-mode EM algorithm for high-resolution 3-D PET image reconstruction into large arrays." Nuclear Science, IEEE Transactions on 49(3): 693-699.

Sendur, L. and I. W. Selesnick (2002). "Bivariate shrinkage with local variance estimation." IEEE Signal Processing Letters 9(12): 438-441.

Shalchian, B., H. Rajabi, et al. (2009). "Assessment of the wavelet transform in reduction of noise from simulated PET images." J Nucl Med Technol 37(4): 223-228.

Shen, R. L. a. Y. (2006). "Noisy Image Segmentation by Modified Snake Model." J. Phys.: Conf. Ser. 48: 369.

Shidahara, M., Y. Ikoma, et al. (2007). "PET kinetic analysis: wavelet denoising of dynamic PET data with application to parametric imaging." Ann Nucl Med 21(7): 379-386.

Shih, Y. Y., J. C. Chen, et al. (2005). "Development of wavelet de-noising technique for PET images." Computerized Medical Imaging and Graphics 29(4): 297-304.

Simoncelli, E. P. (1999). Modelling the joint statistics of images in the wavelet domain. Proc SPIE, 44th Annual Meeting. 
Starck, J. L. (2001). Very High Quality Image Restoration by Combining Wavelets and Curvelets. SPIE conference on Signal and Image Processing: Wavelet Applications in Signal and Image Processing.

Starck, J. L., E. J. Candes, et al. (2002). "The curvelet transform for image denoising." IEEE Trans Image Process 11(6): 670-684.

Starck, J. L., J. Fadili, et al. (2007). "The undecimated wavelet decomposition and its reconstruction." IEEE Trans Image Process 16(2): 297-309.

Starck, J. L., F. Murtagh, et al. (2010). Sparse image and signal processing : wavelets, curvelets, morphological diversity. Cambridge ; New York, Cambridge University Press.

Tixier, F., M. Hatt, et al. (2012). "Reproducibility of tumor uptake heterogeneity characterization through textural feature analysis in 18F-FDG PET imaging." Journal of Nuclear Medicine in press.

Tsoumpas, C., F. E. Turkheimer, et al. (2008). "Study of direct and indirect parametric estimation methods of linear models in dynamic positron emission tomography." Medical Physics 35(4): 1299-1309.

Turkheimer, F. E., R. B. Banati, et al. (2000). "Modeling dynamic PET-SPECT studies in the wavelet domain." Journal of Cerebral Blood Flow and Metabolism 20(5): 879-893.

Turkheimer, F. E., N. Boussion, et al. (2008). "PET image denoising using a synergistic multiresolution analysis of structural (MRI/CT) and functional datasets." J Nucl Med 49(4): 657-666.

Turkheimer, F. E., M. Brett, et al. (1999). "Multiresolution analysis of emission tomography images in the wavelet domain." J Cereb Blood Flow Metab 19(11): 1189-1208.

Webb, A. R. (2003). Introduction to biomedical imaging. Piscataway, NJ

Hoboken, N.J., IEEE Press ;

Wiley.

Zubal, I. G., C. R. Harrell, et al. (1994). "Computerized three-dimensional segmented human anatomy." Med Phys 21(2): 299-302. 
Vitae

Adrien Le Pogam is a post-doc fellow with the National Institute of Health and Medical Sciences (INSERM) in France. He is based within the INSERM Medical Information Processing Laboratory in Brest. He received his engineering degree from the ENIVL School, Blois, France in 2005, and his PhD degree from the University of Tours in 2009. His research interests are image denoising and deconvolution for oncology applications.

Houda Hanzouli is a PhD student with the University of Brest in France under the supervision of Mathieu Hatt and Dimitris Visvikis. She is based within the INSERM Medical Information Processing Laboratory in Brest. She received her Master degree in 2011 from the National School of Computer Sciences (ENSI, Tunis, Tunisia). Her PhD focuses on developments for multiresolution and multimodal medical image processing and analysis.

Mathieu Hatt is a junior research associate with the National Institute of Health and Medical Sciences (INSERM) in France. He is based within the INSERM Medical Information Processing Laboratory in Brest. He received his Master degree in computer sciences from the University of Strasbourg in 2004, and his $\mathrm{PhD}$ degree from the University of Brest in 2008. His research interests are image and information processing and analysis for medical imaging in oncology and radiotherapy clinical applications.

Catherine Cheze-Le Rest is a nuclear medicine physician and a professor at the University of Poitiers, France. She is based within the Academic Nuclear Medicine Department of the Milétrie University Hospital. She received her MD degree and her PhD degree from the University of Brest in 1994 and 2005 respectively. Her research interests are optimal uses of PET imaging in oncology clinical applications.

Dimitris Visvikis is a director of research with the National Institute of Health and Medical Sciences (INSERM) in France. He is based within the INSERM Medical Information Processing Laboratory in Brest, where he is in charge of a group on quantitative multi-modality imaging for diagnosis and therapy in oncology. He obtained his $\mathrm{PhD}$ degree from the University of London in 1996. After that he has worked as a Senior Research Fellow in the Wolfson Brain Imaging Centre of the University of Cambridge and spent five years as a principal physicist in the Institute of Nuclear Medicine in University College London. 


\section{Appendix}

The ridgelet transform $r$ of a signal $s \in L^{3}\left(\mathfrak{R}^{3}\right)$ is defined by :

$$
r(a, b, \theta, \gamma)=\int_{\Re^{3}} \psi_{a, b, \theta, \gamma}(\vec{x}) s(\vec{x}) d(\vec{x})
$$

with

$$
\begin{gathered}
\psi_{a, b, \theta, \gamma}(x)=a^{-1 / 2} \psi\left(w_{\vec{x}, \theta, \gamma}-b / a\right) \\
w_{\vec{x}, \theta, \gamma}=x_{1} \cos (\theta) \cos (\gamma)+x_{2} \cos (\theta) \sin (\gamma)+x_{3} \sin (\theta) \\
\vec{x}\left(x_{1}, x_{2}, x_{3}\right)
\end{gathered}
$$

The ridgelet coefficients are defined in the Radon domain as following:

$$
r(a, b, \theta, \gamma)=\int_{\Re^{3}} \psi_{a, b, \theta, \gamma}(t) R_{s}(t, \theta, \gamma) d(t)
$$

The Radon transform of a signal s is defined by:

$$
R_{s}(t, \theta, \gamma)=\iiint_{\mathfrak{R}^{3}} s(x) \delta\left(\vec{w}_{\vec{x}, \theta, \gamma}-t\right) d \vec{x}
$$

